\title{
Systèmes vivants : lois locales et globales, invariance d'échelle.
}

\section{Living systems : local versus global \& individual versus whole. Emergence \& diversity : local and whole scale invariant laws.}

\author{
Pierre BRICAGE \\ Faculté des Sciences, Université de Pau et des Pays de l'Adour UPPA, Pau, France, http://web.univ-pau.fr/ bricage/ \\ Association Française de Science des Systèmes AFSCET, Paris, France, http://www.afscet.asso.fr \\ World Organisation of Systems and Cybernetics WOSC, Lincoln, UK, http://www.wosc.co/ \\ International Academy for Systems and Cybernetic Sciences IASCYS, Vienna, Austria, http://iascys.org \\ pierre.bricage@univ-pau.fr \\ Association PELLEAS, 9 route de Saint ARMOU, 64450 Lasclaveries, France
}

\begin{abstract}
To survive that is 'to eat and not to be eaten', to live on [9, 11]. Any living system [10], to survive and live on [9], whatever is its spatial [28] and temporal [23, 29, 35] level of organization, owns 7 invariant qualitative characteristics (degrees of freedom) [19]. Any alive system is formed by embedding and juxtapositions [17] of pre-existing systems [22]. How are the local quantitative laws, of their spatial-temporal structuring and functioning, associated with these qualitative characteristics independently from the dimensional scales? How are they independent/dependent from the new global level of organization and the local situations of emergence? How do the local actors become mutually integrated into their global whole? And reversely (systemic constructal law [4]), why and how is the global whole reciprocally integrating the local parceners $[18,20]$ ? At every level of organization, the evolution of the living systems obeys 5 organizing principles of emergence [33] and the space (the volume of the adult system VA) and the duration (time of generation tg) are linked through a power law (generalized Kepler's 3rd law like $\mathrm{V}_{\mathrm{A}}{ }^{2}=\mathrm{C} \cdot \mathrm{tg}^{3}$ ), a law of growth (figure 3) and exchange (figure 4). As all the sub-systems which live in it, the whole Universe is living in an ecoexotope that it can share with other Universes.
\end{abstract}

Résumé- Survivre c'est "manger et ne pas être mangé", "pour se survivre" $[9,11]$. Tout système vivant [10], pour survivre et se survivre [9], quel que soit son niveau spatial [28] et temporel [23, 29, 35] d'organisation, possède 7 caractéristiques qualitatives invariantes (degrés de liberté) [19]. Tout système vivant est formé par emboîtements et juxtapositions [17] de systèmes préexistants [22]. Les lois quantitatives locales de structuration, spatiale et temporelle, et de fonctionnement, associées à ces caractéristiques qualitatives sont-elles indépendantes de l'échelle, c'est-à-dire du nouveau niveau global, d'organisation et d'émergence ? Comment le local s'intègre-t-il au global ? Et réciproquement (loi systémique constructale [4]), comment le global intègre-t-il le local $[18,20]$ ? L'évolution du vivant obéit à 5 principes organisateurs d'émergence présents à tous les niveaux d'organisation : 1- un principe de maintien (d'invariance des fonctionnalités) : sept caractéristiques (degrés de liberté) sont mutuellement nécessaires et suffisantes pour survivre et se survivre, 2- un principe moteur d'évolution (d' “obligation organique“) : l'appartenance à une chaîne alimentaire, "manger et ne pas être mangéc", "tôt ou tard il est impossible de ne pas être mangé", 3- un principe de structuration: d'organisation modulaire (modularité) par emboîtements et juxtapositions de modules pré-existants, 4- un principe de phylogénie (d'escalade de la complexité) : seules survivent et se survivent les associations à avantages et inconvénients réciproques et partagés (ARMSADA), 5- un principe d'ontogénie, d'invariance d'échelle (homothétie fractale), avec une loi de puissance (sorte de 3ème loi de Kepler généralisée) reliant espace (le volume du système adulte) et temps (le cycle de génération).

Key words: "Associations for the Reciprocal and Mutual Sharing of Advantages and DisAdvantages" (ARMSADA http://armsada.eu), loi puissance, loi systémique constructale, phylotagmotaphologie [28], structures fractales [17]

\section{INTRODUCTION}

Tout système comporte 3 entités indissociables qu'il faut considérer ensemble dans leur structure fonctionnelle : un tout, des acteurs, des interactions (des liens entre ces acteurs et avec le tout). On ne peut pas se centrer sur l'une de ces entités en ignorant les autres. Il faut toujours les considérer ensemble (approche holistique) [14, 27, 37] (figure 1). 


\author{
THE GAUGE INVARIANCE OF LIVING SYSTEMS \\ L' INVARIANCE DE JAUGE DES SYSTÈMES VIVANTS
}

\title{
A. To survive that is "to eat and not to be eaten" - Survivre c'est "Manger et ne pas être mangé". \\ Every Living system is integrated into a food chain - Tout système vivant appartient à une chaîne alimentaire.
}

Les lichens sont des organismes issus de l'association d'une algue et d'un champignon. En 1879, De Bary introduisit la notion de symbiose, "association à bénéfices mutuels", pour désigner cette union. Depuis, cette conception "inexacte” a perduré [12]. L'algue, capable de photosynthèse, capable de fabriquer sa matière organique en l'absence de matière organique préexistante, en l'absence de toute forme de vie, est la partie chargée de la production. Le champignon, habituellement saprophyte, consommateur, est incapable de fabriquer sa matière organique. Abri riche en eau et en sels minéraux, le champignon en apparence "offre" à l'algue le gîte et le couvert. En réalité, il "cultive" l'algue en son sein, comme l'homme "élève" ses animaux domestiques, et en retour de cet "investissement", par des filaments suçoirs, le champignon "mange" les cellules de l'algue... L'algue, "agressée", montre des symptômes de "souffrance" métabolique, et des zones de mort en masse par nécrose. En fait, les deux partenaires se nourrissent et se contrôlent réciproquement. Le partenaire champignon, comme la partie racinaire des plantes supérieures, puise "la sève brute". Le partenaire algue, comme la partie foliaire, élabore "la sève élaborée". Fonctionnant à la fois comme un végétal et un animal, le lichen représente un niveau d'organisation plus élevé que ceux de l'algue et du champignon. C'est un nouvel organisme, car il élabore des molécules qu'aucun autre champignon ou qu'aucune autre algue ne peuvent élaborer. C'est aussi un écosystème, contenant une chaîne alimentaire. Tout système vivant est le siège d'un flux de matière et d'énergie. Survivre c'est "Manger et ne pas être mangé", "tôt ou tard, il est "impossible de ne pas être mangé" [9, 10,11]. La survie d'un écosystème dépend d'au moins une espèce clé-de-voûte dont la disparition entraîne celle de l'écosystème, de la chaîne alimentaire à laquelle elle appartient. L'espèce humaine est l'espèce clé-de-voûte d'anthroposystèmes comme les agro-systèmes [31, 32].

$\mathrm{Au}$ sein du Lichen, l'algue élabore des vitamines qui contrôlent la croissance du champignon, et le champignon élabore des substances antibiotiques qui contrôlent la croissance de l'algue. Chacun des organismes, anciennement libres, mais maintenant indissociables, juxtaposés et emboîtés au sein du Lichen, est une espèce clé-de-voûte pour l'autre. Au laboratoire, privé de l'algue, le champignon ne peut survivre et croître que sur un mode désordonné et indifférencié, sans acquérir la capacité de se survivre. La capacité de croissance, en masse ou en nombre, n'est pas une fin en soi. La croissance n'a d'intérêt que parce qu'elle permet d'acquérir une masse/un nombre critique qui rend possible la mise en place d'un développement, durable si la croissance se stabilise. La croissance ne permet pas l'acquisition de capacités nouvelles, elle n'en est que le préalable indispensable : “la croissance est le préalable au développement“ $[8,14]$.La survie de l'organisme du lichen est fondée sur cet état d'équilibre entre les deux partenaires. Pour survivre, le partenaire champignon doit limiter son agression sur l'associé algue. Hôte hébergeant, et habité, il paie un double coût : le coût de l'hébergement de l'algue et le coût d'une croissance limitée par celle de l'algue. Pour que le champignon survive il faut d'abord que l'algue survive. L'algue, hôte hébergé (et captif !) paie, lui aussi, un double coût : le coût de la survie de sa population de cellules, qui passe par la non-survie d'une partie des individus (les cellules qui sont mangées ${ }^{1}$ ), et, le coût d'une croissance limitée par la croissance du champignon (elle-même limitée par celle de l'algue). Les inconvénients pour les deux partenaires sont énormes, ils restent "nains". Et, si l'un meurt, l'autre meurt. Les 2 partenaires totalement solidaires ne forment qu'un. Les inconvénients pour l'un sont des avantages pour l'autre et réciproquement, il n'y a de bénéfice que pour le Tout. C'est une Association à Avantages et Inconvénients Réciproques et Partagés (ARMSADA). Un nouveau système, c'est à dire, à la fois une forme nouvelle d'organisation pour survivre et se survivre (endophysiotope) et une forme nouvelle d'intégration au milieu externe de survie (écoexotope), est né, avec un changement d'échelle temporelle. Le système peut survivre des siècles ! On connaît plus de 20.000 espèces de lichens. Ils forment la première végétation, en altitude et vers les pôles, à la limite des neiges et des glaces permanentes, et sur les substrats secs ou salés, organismes pionniers, ils survivent dans des milieux où la capacité d'accueil de l'écoexotope est nulle pour la capacité d'être accueilli de l'endophysiotope de chacun des partenaires isolément, mais suffisante pour l'endophysiotope du Lichen. Ils possèdent la capacité extraordinaire de passage à l'état de vie ralentie, puis de reviviscence. Ils colonisent les sols nus, les écorces, les rochers, les murs, le verre, les rambardes d'autoroute...

Végétaux pionniers, ils permettent l'installation de nouvelles formes de vie animale... qui les mangent !

1 Mais de toute façon la durée de survie d'une cellule, quelle qu'elle soit, est limitée. 


\section{B. The 7 degrees of freedom of all living forms - Les 7 degrés de liberté de tout système vivant.}

Pour survivre et se survivre, tout système vivant, quel que soit son niveau d'organisation, doit posséder 7 capacités fonctionnelles mutuellement nécessaires et suffisantes [11, 18]. La capacité de mobilisation de la matière et de l'énergie, la capacité de réaction à des stimulations [7], la capacité de croissance, la capacité d'intégration à un écoexotope de survie [6], la capacité de mettre en place et de maintenir une organisation interne (endophysiotope) [8], la capacité de mouvement lui permettent de survivre [9] et de mettre en place la capacité de se survivre, caractéristique du stade adulte, qui permet à son espèce, à sa forme de vie, de survivre. Ces 7 caractéristiques sont mutuellement nécessaires et suffisantes pour définir tout niveau d'organisation du vivant. C'est l'invariance de jauge du vivant [10]. Mais se survivre représente un coût d'organisation et d'intégration pour la survie de tout organisme. ${ }^{2}$ Se survivre est aléatoire et n'est possible qu'un temps [11,12,13,14]. Comment augmenter durablement la capacité d'accueil de son écoexotope de survie ou la capacité d'être accueilli de son endophysiotope, pour survivre et se survivre ? Comment s'affranchir, au moins momentanément, des contraintes d'espace et de temps, "être au bon endroit au bon moment" ? Comment échapper, au moins momentanément, à la violence de la lutte pour la vie, "survivre c'est manger et ne pas être mangé, pour se survivre" ? ... en mettant en place des ARMSADAs!

\section{Associations for the Reciprocal and Mutual Sharing of Advantages and DisAdvantages (ARMSADA): THE ONLY WAY TO SURVIVE FOR LIVING SYSTEMS IS TO MERGE INTO AN ARMSADA.}

La biosphère terrestre appartient aux Monères. Toutes les cellules (niveau d'organisation $\boldsymbol{i}$ ) des organismes méta-cellulaires eucaryotes (niveau $\boldsymbol{i}+\boldsymbol{1}$ ) proviennent de la fusion d'organismes bactériens (niveau $\boldsymbol{i}-\boldsymbol{1}$ des Monères) [21]. Même le placenta humain est habité par une communauté d'espèces bactériennes, réduite en nombre mais plus variée en espèces que celles qui habitent l'intestin, le vagin, la bouche ou la peau et qui ressemble à celle de la cavité buccale [1]. Ce profil de bactéries est très stable d'une femme à une autre. Par leur présence elles contribuent à la synthèse de vitamines et de cofacteurs enzymatiques indispensables à la survie du couple mère-foetus. Elles permettent la mise en place du système immunitaire propre au foetus. ${ }^{3}$

\section{Every Living Systems is both an EcoSystem and an EcoSystem of EcoSystems.}

Une observation au microscope électronique de l'endophysiotope d'une cellule végétale chlorophyllienne met en évidence plusieurs compartiments fonctionnels, juxtaposés et emboîtés : le cytoplasme, les vacuoles, les mitochondries, les chloroplastes, les peroxysomes. La cellule (niveau $\boldsymbol{i}$ ) représente un niveau d'organisation plus élevé que celui (niveau $\boldsymbol{i}-\mathbf{1}$ ) du cytoplasme, des mitochondries et des chloroplastes, qui la constituent, qui l'habitent, et pour l'endophysiotope desquels elle représente le nouvel écoexotope de survie. Elle exprime un nouveau mode d'intégration au milieu de survie. Les compartiments ne sont pas disposés de façon aléatoire dans l'espace de survie intra-cellulaire. Le cytoplasme, lieu de protection, d'échanges et de communication, englobe tous les autres. La vacuole, lieu de réserve d'eau, est au contact des mitochondries, qui produisent de l'eau (déchet respiratoire), et des chloroplastes, qui consomment de l'eau (matière première de la photosynthèse). L'aliment des uns est le déchet des autres. Les mitochondries, qui consomment de l'oxygène et des sucres (aliments respiratoires), sont au contact des chloroplastes, qui eux produisent des sucres et de l'oxygène (déchet de la photosynthèse). Le déchet des uns est l'aliment des autres. Les mitochondries descendent génétiquement d'anciennes bactéries, autrefois à vie libre. Elles ont "colonisé" le cytoplasme, du système ancestral d'accueil, à l'origine des cellules eucaryotes. Ainsi s'est mise en place une association à avantages et inconvénients réciproques et partagés dans laquelle les mitochondries éliminent l'oxygène qui serait toxique, en leur absence, pour le cytoplasme. En contrepartie, le cytoplasme héberge, protège et nourrit les mitochondries. Au cours de leurs fonctionnement ago-antagonistes, chloroplastes et mitochondries produisent des superoxydes, extrêmement toxiques, qui sont recyclés en substances utiles par les peroxysomes, pour le bénéfice de chacun et du Tout, la cellule $[8,10,11,22,33]$.

2 Chez l'araignée, la capacité de "se survivre" réduit la capacité de "survivre" [58].

La reproduction -la capacité de se survivre- a un coût, payé par la croissance, qui diminue la capacité de survivre.

3 The human placental microbiome species and man species endophysiotope have coevolved as a functional ecosystem composed of distinct body site niches (microbiome ecoexotopes) with metabolic and antigenic diversity. Intracellular bacteria with diverse metabolic and immune regulatory functions from different taxa and with various genes heritages are inhabiting human body site niches as the oral, skin, nasal, vaginal, and gut. Compared with those from non-pregnant women, the unique placental microbiome niche is composed of nonpathogenic commensal microbiota. The placental microbiome profiles is most akin to the human oral one. Taxonomic unit analyses revealed associations of the placental microbiome with a remote history of antenatal infection such as urinary tract infection in the first trimester, as well as with preterm birth [Adapted from 1]. 


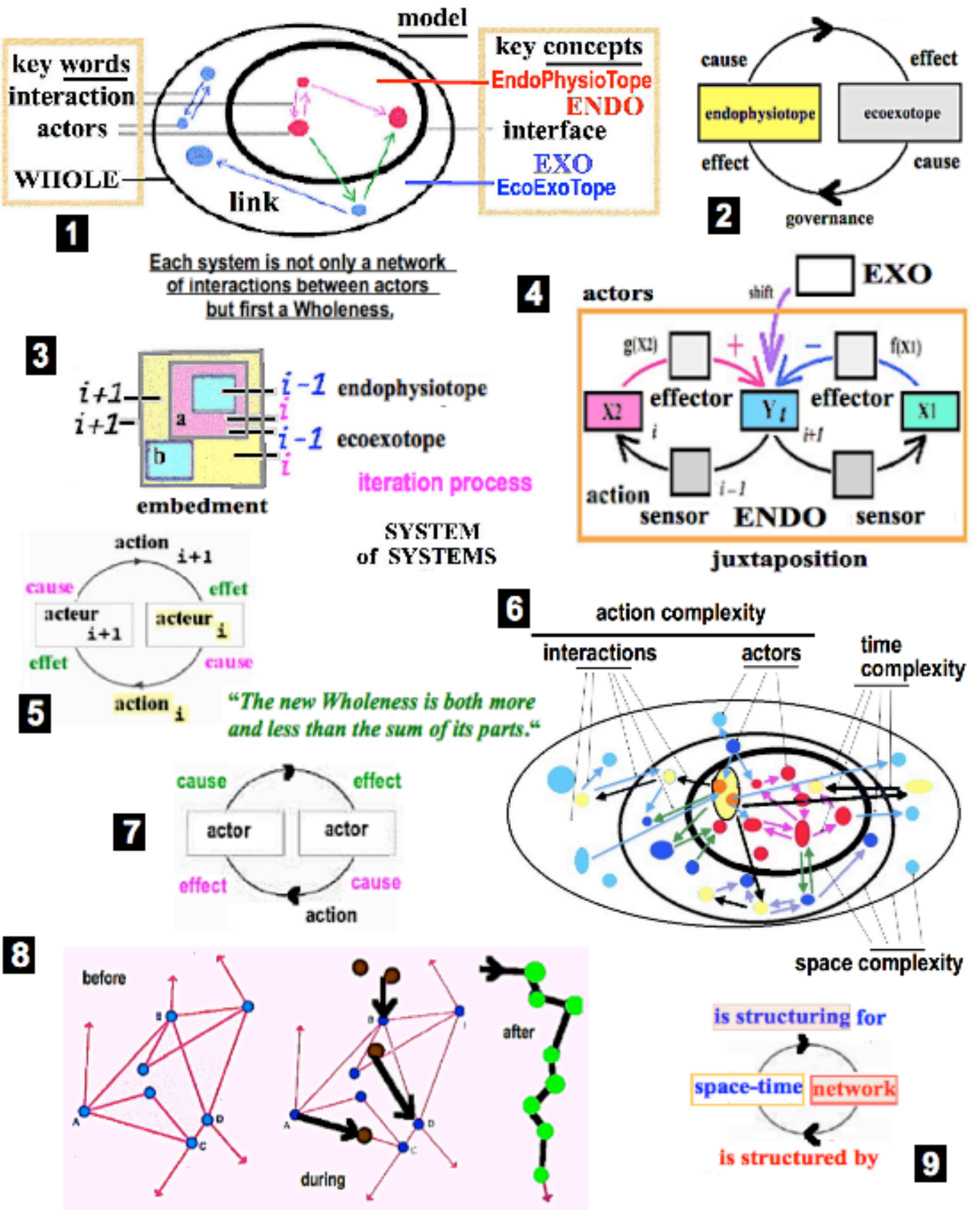

Figure 1. Systemics of living system of systems. 
Figure 1. Systemics of living system of systems [14, 28, 29, 36, 37, 39 http://www.wccs14.org/]

1. integration \& organization (model \& keywords). A system is always made of 3 kinds of entities: actors, interactions, a Whole. Every living system whatever its level of organization, is integrated into an ecoexotope (EXO: external, tope: space-time, eco: of inhabitation) of survival which gives the system's endophysiotope (ENDO: internal, tope: space-time, physio: of functioning) an hosting capacity.

2. "interaction is integration and integration is interaction. "EXO hosting capacity changes result in ENDO capacity to be hosted changes, and mutually. Each living system-of-systems is integrated into an ecoexotope within which it is more adapted to the interactions network than other systems-of-systems are $[6,7]$. Systemic Constructal Law : each effect has a cause and each effect is a new cause for a new effect. Climate changes, communities changes of the ecoexotope and endophysiotope changes are overlapping and in a loop. The changes of the ecoexotope hosting capacity, in quality or in quantity, particularly due to recycling, are controlling the growth and constrained changes in the functional, spatial and temporal organization of the capacity to be hosted $[6,7,8]$. That feedback is controlling the growth and the development $[31,32]$ : (hosting capacity) $x$ (capacity to be hosted) $=k$. Step by step, by cycling through amplifying loops, "a threshold of growth is a requisite for development, a threshold of development is a requisite for growth".

3. emergence and organization. During the life evolution process, new endophysiotopes of living systems are emerging through an iteration process of embedment and juxtaposition of pre-existing systems. Each living system is a system-of-systems. An endophysiotope of a $\boldsymbol{i}$ level of organization is the ecoexotope of survival of an endophysiotope of $\boldsymbol{i}-\boldsymbol{1}$ level and, mutually, a $\boldsymbol{i}$ 1 level ecoexotope is integrated into a $\boldsymbol{i}$ level endophysiotope. That allows the emergence, with an effective great diversity, of new modular blueprints.

4. flows of matter, energy \& information. Even if they are specialized as effector -responding to an appropriate, external or internal, stimulus by a specific response (a movement, a secretion,...)- or as sensor -summing, external or internal, stimuli-, actors are acting together directly on the others and indirectly on themselves, within and between levels of organization because actors' actions are both causes and effects (see below $\mathbf{5} . \&$ 7.).

5. actors' actions are both causes and effects between organization levels. The juxtapositions and embedments of the parts into their Whole are known but the functioning of the Whole is not predictable from the functioning of the parts [8, 9, 11, 12], so, obviously, "a whole is both more and less than the sum of its parts.". The modularity of the actors and interactions is the source of exaptations $[14,19]$. And we have to define the parameters of this complexity (see 6.).

6. parameters for the qualification and quantification of complexity. We must first qualitatively identified and quantitatively characterized all the system's parts, by deconstructing it (reductionism). But through reconstructing the whole from the parts (holism) we cannot explain new properties (emergence) only from the ancient ones [31, 38]. So we have to define 3 parameters for measuring the system's complexity: the Action complexity which is given both by the numbers of each kind of actors (a colored "point" for each type) -all numbers will give the total actors number (we can also define actors quantitatively by the surface of the point)- and the numbers of each kind of interactions (a colored arrow for each kind) -which all will give the total interactions number (we can also define interactions quantitatively by the thickness of the arrow)-, the Time complexity which is given by the duration of all the interactions that take place during a period (a time cycle of survival) [35] and the Spatial complexity which is given by the absolute and relative surface limits (interfaces) of the ecoexotope (EXO) and endophysiotope (ENDO) [20]. The emergence of an ARMSADA depends on the ecological, economical and genetical history of every actor, on its place into the system, on the global context of interaction and on its local fate (see 8. : percolation).

7. actor's actions are both causes and effects within a level of organization. An action (from Latin, "accium" by 1114 and "agere", to act, by 1310) is the effect of all the strengths exercised by an actor (or a system) on an other one. Every actor (of a system) is an "entity endowed with potentialities of actions", that may exercise an action on another actor (of the same system or another one). The actor is the initiator of the action (that transforms, modifies), the cause which produces an effect. This effect (the answer) is measurable, qualitatively or quantitatively. The action can allow to pass from a state to an other one, only if the supplied and received stimulation, is enough, beyond a threshold of intensity. The effect produced by the initial cause becomes a cause of a new effect back on the initial cause (5. \& 6.): systemic constructal law.

8. percolation process leading to emergence of a new system of cooperating interactions.

The stability and resiliency of a system, while facing to changes of its endophysiotope and ecoexotope, is depending on the number of actors and the percolation process of their interactions. Emergence is always a spatial and temporal, structural and functional, metamorphosis within a periodic cycling equal sharing network $[21,22,23,24,33,34]$. There are always 3 simultaneous processes in a metamorphosis: -lysis of ancient structures with the disappearance of previous actors during the interactive process of integration of at least 1 new actor, -creation of new functional structures, new actors that were not there before, are integrated into "the coming network", -ancient actors are conserved but "transformed" in their action, or in their place, or in their time of action. The ARMSADA inter-merging process, making from different species a new one, by embedment and juxtaposition (3. \& 4.), is an intra-emerging synchronizing one, a percolation extra-emerging process with threshold and delay, like the timing mechanism that is involved in intra-organism differentiation at the cell level. Time is very important but, soon or late, only small structural changes in space may be sufficient to trigger the reciprocal interactions that allow the emergence of an ARMSADA. Integration is depending both on the actors' age and stage, their interactions and the Whole. Connectedness in a network often shows a threshold behavior. At some point, the addition of a just a few more connections can cause a substantial fraction of the network to be connected $[22,24]$. 
9. the space-time of the whole is structured by the network of interactions of its actors. The endophysiotope of a $i$ level of organization is the ecoexotope of previous $\boldsymbol{i}-\boldsymbol{n}$ levels. Due to the parceners' half-autonomy, abilities of the previous levels are lost while simultaneously new ones are gained. "A space for each one and each one in its space" and "A time for each one and each one in its time". Through the iteration of the process of ARMSADAs' emerging, each new more-and-more complex "system-ofsystems" is more-and-more independent of its ecoexotope (http://tinyurl.com/phylotagmotaphology). In their new shared endophysiotope the partners are all spatially and temporally interdependent (figure 3a).

Comme dans le Lichen, pour que l'un survive il faut d'abord que l'autre survive, et réciproquement; il n'y a de bénéfice que pour le Tout. Si la mitochondrie, ou le noyau, meurt à la suite d'une infection virale par exemple, la cellule meurt, par apoptose ou nécrose [33, 34].

\section{More increasing biodiversity through more ARMSADA emergences.}

Association à avantages et inconvénients réciproques et partagés entre partenaires agoantagonistes, la symbiose est une organisation durable à des échelles de temps et d'espace très supérieures à celles des partenaires constituant l'association. L'avantage est énorme pour l'association. Mais le coût peut être énorme pour les partenaires. Mais, même s'il se paie d'un coût énorme, tout inconvénient peut être supporté, s'il crée un avantage inestimable, le seul pour lequel il vaille de survivre, même difficilement, celui de se survivre.

Dans tout écosystème, Il existe une relation entre la biodiversité et la densité. La biodiversité est un moyen de faire face aux changements. Plusieurs niveaux de biodiversité peuvent aboutir à la mise en place d'un équilibre dynamique de survie optimale $[29,31]$. Comme les niveaux d'équilibre sont imprévisibles, tout ce qui change la biodiversité entraîne un déséquilibre [20,26]. Tôt ou tard tout organisme vivant devient une proie pour des prédateurs, nouveaux ou anciens. Survivre c'est "Manger et ne pas être mangé". "Tôt ou tard, il est "impossible de ne pas être mangé". C'est la mise en place de nouvelles ARMSADAs qui, avec l'apparition de nouveaux plans d'organisation, permet l'accroissement de la biodiversité, tout en conservant la biodiversité pré-existante [57].

\section{LES “DIMENSIONS“" DES ESPACE-TEMPS-ACTIONS DES SYSTĖMES VIVANTS. ${ }^{4}$}

$\mathrm{Au}$ sein du réseau internet, la dépendance temporelle de la connectivité et l'optimisation de la structure connective suivent une loi de puissance..$^{5}$ Au sein d'un réseau neuronal [48], la distribution du volume de l'arborisation terminale axonique (nombre de ramifications) est corrélée négativement à la longueur de l'axone (en micromètre) selon une loi de puissance d'exposant $\mathbf{- 2 , 2}$. C'est l'inverse pour la densité des dendrites. Au niveau d'organisation cellulaire (niveau $\boldsymbol{i}$ ), les réseaux d'interactions, entre protéines (niveau $\boldsymbol{i}$-2) et entre compartiments intra-cellulaires (niveau $\boldsymbol{i}$-1), sont des réseaux hiérarchisés obéissant à des lois de puissance [29]. ${ }^{6}$ Est-ce aussi le cas aux autres niveaux d'organisation (figures 2 \& 3) ? Quelles sont les "dimensions", physiques et physiologiques, correspondant aux "capacités caractéristiques" (figures $2 \& 4$ ) des systèmes vivants ?

\section{E. La DIMENSION TEMPORELLE aux différents niveaux d'organisation: TIME power laws.}

La réponse à une stimulation d'un neurone (niveau $i$ ) n'est possible, que si le produit (intensité)x(durée), caractéristique du stimulus, dépasse un seuil d'excitation. Puis il passe par un état de non-excitabilité, même si la stimulation est supérieure au seuil, avant de pouvoir répondre à nouveau. Cet état réfractaire disparait après un temps de restauration. Le temps d'adaptation à un stimulus (en ms) et le temps de restauration sont inversement proportionnelles (loi hyperbolique) $)^{7}$ et sont liés au stimulus par une loi de puissance ${ }^{8}$ [48].

Le temps de restauration d'une cellule (niveau $\boldsymbol{i}$ ), en ms ou en s, ou d'un organisme (niveau $\boldsymbol{i}+\boldsymbol{1}$ ), en s ou en min, peut être réduit par des substances anti-oxydantes (niveau $\boldsymbol{i}-\boldsymbol{Z}$ ), en concentrations micromolaires. La durée de restauration $\boldsymbol{y}$ et la concentration de l'effecteur protecteur $\mathbf{x}$ sont reliées par une loi de puissance d'exposant $+\mathbf{1} .{ }^{9}$

4 Les références sont accessibles en données complémentaires (supplementary data), avec la présentation orale (slides), à http://web.univ-pau.fr/ bricage/files/bricage s11-sd-paper.pdf

5 Power law distribution of the fitness of the network

6 At the cell organization level (level $\boldsymbol{i}$ ), many protein interaction networks (level $\boldsymbol{i}-\boldsymbol{2})$ and metabolic networks between cell compartments (level $\boldsymbol{i}-1)$ exhibit an emerging pattern [51] so that the distribution of the connections number of a protein or metabolite follows a power law [3].

7 (adaptation time) $x($ recovery time $)=$ constant, $t 1 x t 2=C$

8 With $\mathbf{y}$ "adaptation time" or recovery time and $\mathbf{x}$ "tilt", graphic form: $\log (\mathbf{y})=\mathbf{k} \cdot \log (\mathbf{x})$

$9 x y=C$ 
Chez les mammifères (niveau $\boldsymbol{i}+\mathbf{1}$ ), la longévité (Maximum LifeSpan, en années) et la masse corporelle (en $\mathrm{kg}$ ) sont liées par une loi de puissance d'exposant $+\mathbf{1} / \mathbf{5}(0,21)$. Chez la jeune fille, l'âge de la puberté, âge d'acquisition de la capacité de se survivre, de passage à l'état adulte (développement), n'est atteint qu'après l'acquisition d'une masse corporelle minimale, pendant la phase de croissance. Si le poids redescend au-dessous de ce seuil l'organisme femelle devient aménorrhé et perd, au moins provisoirement, tant que la masse est insuffisante, la capacité de se survivre [5]. Chez les poissons téléostéens et chez les oiseaux, l'effectif du harem de femelles d'un mâle est corrélé au rapport des dimensions des 2 sexes selon une loi de puissance $+\mathbf{2}$. Les plus gros mâles, relativement aux tailles des femelles, ont des plus grands harems. ${ }^{10}$

La reproduction a un coût qui est "payé" par la croissance.

"L'atteinte d'un seuil prend du temps. ", "La croissance permet de changer d'espace-temps. “

Les activités de populations d'une même chaîne alimentaire sont souvent synchronisées. ${ }^{11}$

Chez les bactéries (niveau $\boldsymbol{i}-\mathbf{2}$ ), la croissance, représentée par une courbe sigmoïde ou logistique, suit une loi exponentielle. La "durée de survie" et le "temps de génération" (la durée minimale de la phase de croissance nécessaire et suffisante pour qu'une bactérie se divise) sont liés par une loi puissance. Chez les Monères (bactéries et archées), le temps de génération et le décodage de l'information génétique (les biais dans l'usage des codons) suit une loi de puissance $\mathbf{- 2 / 3}$. Chez les mammifères et les oiseaux, le temps de génération est co-relié à l'entropie selon une loi de puissance $+\mathbf{1}(0,91)$ et l'entropie est corrélée à la masse selon une puissance $+\mathbf{1 / 3}(0,27)$. La durée maximale de survie est co-reliée à l'entropie selon une loi de puissance $+\mathbf{1}(1,0)$.

Les phénomènes climatiques terrestres (niveau macroscopique $\boldsymbol{i}+\boldsymbol{3}$, biosphère terrestre) obéissent à une loi de puissance. La "durée de vie" d'un phénomène (en années terrestres) est proportionnelle à la puissance $+\mathbf{5} / \mathbf{3}$ de sa dimension (rayon en $\mathbf{k m}$ ). La "durée de survie" (en années humaines) d'une institution internationale (niveau macroscopique $i+1$, espèce humaine), gouvernementale ou non, est proportionnelle à la puissance $+\mathbf{1}$ du nombre de ses membres. La durée peut donc être reliée à la croissance en masse ou en taille, et à la croissance en nombre.

Depuis l'apparition du SIDA vers 1980, le nombre cumulé des cas de l'épidémie humaine (niveau $\boldsymbol{i}+\mathbf{1}$ ), en augmentation, $\underline{\text { la croissance en nombre }}$, est lié(e) à la durée par une loi de puissance.

\section{F. La DIMENSION SPATIALE aux différents niveaux d'organisation : SPACE power laws.}

Chez Plasmodium falciparum, organisme parasite responsable du paludisme chez l'homme (niveau $i+1$ ), l'architecture du génome (la distance génomique) est corrélée à la probabilité d'expression des gènes (niveau $\boldsymbol{i}-\boldsymbol{2}$ ) selon une loi de puissance -1. L'espace est organisé structurellement et fonctionnellement selon une loi de puissance. Chez tous les organismes vivants, des virus aux plantes supérieures, le nombre de protéines (le nombre de gènes codant pour au moins une protéine ${ }^{12}$ ) est corrélé à la taille du génome, en Méga-bases nucléiques (la longueur de l'information potentielle) selon une loi de puissance dont l'exposant décroissant, dépend du niveau d'organisation : +2 pour les virus aux génomes les plus réduits, $+\mathbf{1}$ pour les virus et monères (bactéries, archées) (niveau $i-1),+1 / 2$ pour les eucaryotes cellulaires primitifs (niveau $\boldsymbol{i}$ ) à $\mathbf{+ 1 / 4}$ pour les eucaryotes méta-cellulaires (niveau $\boldsymbol{i}+\mathbf{1}$ ) plus évolués. L'organisation structurale de l'espace-temps est telle que le nombre de protéines (le nombre d'acteurs indispensables à la survie du Tout) tend vers une limite. Ce qui augmente c'est la complexité des phénomènes, et des structures, d'accès, de mise en activité, d'interactions, de régulation, entre les acteurs, locaux, juxtaposés et emboîtés. Chez les mammifères (niveau $i+1$ ), adultes, en fin de croissance, le volume du cerveau est corrélé à la masse (et au volume) du corps selon une loi de puissance de coefficient +2 . La masse d'une étoile (niveau $i+4$ d'un système stellaire), en masse solaire, qui résulte de sa croissance locale par accrétion, est corrélée à la vitesse de son mouvement de "fuite" (en $\mathrm{km} / \mathrm{s}$ ), qui résulte du mouvement de croissance globale de l'Univers (niveau $\boldsymbol{i}+\mathbf{7}$ ), selon une loi de puissance $+\mathbf{5}$.

La croissance dans l'espace et la croissance de l'espace sont liées.

Le nombre d'espèces (niveaux $\boldsymbol{i}-\mathbf{1} \grave{a} \boldsymbol{i}+1$ ) habitant, partageant, le même écosystème (niveau $\boldsymbol{i}+\boldsymbol{2}$ ) est une loi de puissance de la surface disponible. Chez les vertébrés supérieurs (niveaux $\boldsymbol{i}+\mathbf{1}$ ) le coefficient de puissance est de $+\mathbf{1}$ pour les mammifères et de $+\mathbf{1} / \mathbf{2}$ pour les oiseaux. Les oiseaux sont plus indépendants que les mammifères de leur écoexotope de survie. Leur endophysiotope possède une meilleure capacité d'être accueilli.

Les lois de puissance sont représentatives de structures fonctionnelle fractales.

10 In Teleost fishes, average harem size versus male/female body length ratio, patterns of parental investment and sexual selection are depending on mass growth [65].

11 synchronisation d'une population d'hôtes par un virus [68]

12 ORF "Open Reading Frame" 
Un mécanisme physique comme l'Agrégation Limitée par Diffusion (DLA) permet d'aboutir à une structure arborescente, dendritique, sur plusieurs échelles de dimension spatiale. Un dépôt électrostatique, une décharge électrique ou l'injection forcée d'un fluide, dessinent un réseau dont le nombre des points est une loi de puissance proche de $\mathbf{+ 1 , 7}$ (entre $\mathbf{5 / 3}$ et $\mathbf{7 / 4}, 5 / 3=1,6$ et $7 / 4=1,75$ ) du rayon dimensionnel de l'arborescence. Les réseaux de dimension fractale (fractales laplaciennes) sont fréquents dans les systèmes biologiques. Au niveau de l'Univers entier (niveau supposé $i+7$ ), la distance, en $\mathrm{cm}$, sur 25 puissances de 10, est corrélée au temps écoulé depuis le big-bang (quantum d'espace-temps de Planck, niveau supposé $\boldsymbol{i}-9$ ), en s, sur 40 puissances de 10, selon une loi de puissance d'exposant compris entre $\mathbf{3 / 5}$ et $\mathbf{5 / 8}$ (indiscernables... $0,60<0,61<0,62$ ). Encore faut-il distinguer dans cette croissance en volume, la période initiale d'inflation et celle de l'expansion, de la croissance ultérieure, actuelle.

\section{G. La DIMENSION METABOLIQUE aux différents niveaux d'organisation : METABOLIC power laws.}

L'échelle de mesure du pH est, par définition, une échelle logarithmique. Chez les Monères (niveau i-1), les réactions métaboliques de dégradation (catabolisme) ou de synthèse (anabolisme) sont corrélées à l'échelle des $\mathrm{pH}$ selon des lois de puissance, positive ou négative. La vitesse de dégradation est souvent corrélée à la concentration en substrat(s) par une loi de puissance $+\mathbf{1}$ (mais l'exposant peut varier de $\mathbf{- 2}$ à $+\mathbf{2}$ selon les réactions).

\section{" $L$ 'utilisation des aliments disponibles ou des déchets à recycler s'effectue en flux tendus. " 13}

Au niveau cellulaire (niveau $\boldsymbol{i}$ ), pour ce qui est du métabolisme de l'ADN, le processus de traduction (recopie utilisable de l'information utile) est indépendant du métabolisme et de la taille du génome (loi de puissance de coefficient proche de $\mathbf{0}:+0,08)$. Relativement à la taille du génome, le processus de réplication (duplication de toute l'information) obéit à une loi de puissance $+0,41$ (2/5), le processus de (contrôle et) réparation à une loi de puissance $+0,61(\mathbf{3} / \mathbf{5})$, le processus de transcription (synthèse des protéines) à une loi de puissance $+1,94(\mathbf{1 0 / 5})$ et le processus de recombinaison génétique (remaniements de l'information) à une loi de puissance $+2,23(\mathbf{1 1 / 5}){ }^{14}$

Pour ce qui est de l'ensemble des voies métaboliques ${ }^{15}$, par catégorisation fonctionnelle, et relativement à la taille du génome, le métabolisme des sucres obéit à une loi de puissance $+1,10$ alors que les processus métaboliques de base obéissent à une loi de puissance en $+0,80$. Mais le métabolisme des porphyrines suit une loi de puissance en $+1,60$. Les processus de transport et de sécrétion obéissent à une loi de puissance respectivement en $+1,30$ et $+2,00$. Globalement, la mobilisation de la matière et de l'énergie suit une loi globale en moyenne de puissance $\mathbf{3 / 2}(+1,50)$. On pourrait aussi bien dire que les fonctionnalités métaboliques "imposent" une organisation fonctionnelle du génome suivant une loi de puissance $2 / 3$ puisque $2 / 3=1 /(3 / 2)$.

La loi en puissance $+\mathbf{3} / \mathbf{4}$, énoncée par Kleiber en 1932, et utilisée pour décrire la relation entre la taille (la variable présumée $\mathbf{x}$, la cause supposée) d'un organisme méta-cellulaire vertébré (niveau $i+1$ ) et son métabolisme basal (l'effet mesuré $y$, graphiquement $\log (y)=k_{1} \log (x)$ avec $\left.k=3 / 4\right)$, a été enseignée et appliquée pendant des dizaines d'années, expliquée théoriquement en 1997 et étendue à toutes formes de vie en 2000. Mais elle est fausse [45]. Le "bon" exposant n'est pas $+3 / 4(=0,75)$ mais $+2 / 3(=0,66) !^{16}$ En 1947 Kleiber avait proposé expérimentalement, avec la production de chaleur en kcal par jour (ou par h) et la masse en $\mathrm{kg}$, pour les mammifères, une valeur encore plus élevée de $\mathbf{5 / 6}(=0,83)$. De fait, la relation entre le taux métabolique (en watts) et la masse (en g), chez les insectes, isolés ou coloniaux, pour l'individu ou la colonie, donne une pente de $0,82 \pm 0,01$ (soit 5/6). Chez la Daphnie (Daphnia magna, crustacé microscopique), l'intensité respiratoire (la consommation d'oxygène pour le métabolisme, en microlitre/heure) est reliée au poids sec (masse en microgramme) de l'animal (niveau $\boldsymbol{i}+\mathbf{1}$ ) par une loi de puissance d'exposant $+1(\mathbf{6} / \mathbf{6})$ et non $3 / 4 .^{17}$

13 flot, flux (racine indo-européenne, du francique "fluod" et du latin "fluctuare" vers 1140) : à la fois un regroupement et un mouvement, d'objets, d'acteurs, d'actions, d'informations, de matière, d'énergie, effectués par un acteur (ou un système) créateur d'une structure voire d'une organisation. Tout flux implique un travail, une dégradation de l'énergie dans son espace-temps. Dissipant autant, ou plus, d'énergie qu'il n'en incorpore, le système doit posséder une capacité à la reconstitution et au renouvellement, une aptitude à la production d'énergie.

14 Aux incertitudes expérimentales près, qui sont de l'ordre de $5 \%$.

15 Les incertitudes expérimentales sont deux fois plus élevées, elles peuvent atteindre $10 \%$.

16 The $\mathbf{3} / \mathbf{4}$ power law of metabolism, used to describe the relationship between the size of animal meta-cell organisms (niveau $i+1)$ and their resting metabolism proposed by Max Kleiber in 1932, printed in biology textbooks for decades, explained theoretically in 1997 and extended to all life forms in $2000 \ldots$ is wrong ! [45]. Not 3/4 but 2/3 ! That is just the true exponent that describes "the ratio of surface area to volume in a sphere", which surface increases as the square (2D) of the radius and volume as the cube (3D).

The point of resting metabolism is to keep the body warm (and alive !) with the lowest necessary energy use, thus both geometry and common sense suggest this lower rate of $+2 / 3$. A no-spherical system, with more surface area raletive to its volume, will lose heat faster and consume more energy. But the $3 / 4(=0.75)$ and $2 / 3(=0.66)$ slopes are very close to each other. And statistical analysis is very tricky with a lot of uncertainty in the parameter estimates (figure 5). It is more easy to distinguish $3 / 2(=1.50)$ from $4 / 3(=1.33)$.

17 Body size of organisms (from the microscopic level $i-1$ to the macroscopic one $i+1$ ) spans 24 orders of magnitude, but metabolic rate and life span present comparable differences across species. Researchers are explaining this variation in terms of evolutionary entropy, a 
L'objet du métabolisme basal est une production d'énergie minimale pour maintenir une température du corps suffisante pour qu'il reste vivant et fonctionnel au repos. Le coefficient $+2 / 3$ est cohérent avec un flux optimal d'échanges entre l'écoexotope et l'endophysiotope puisque le rapport de la surface rapportée au volume d'une sphère est de $\mathbf{2 / 3}$. La surface (2D) augmentant comme le carré (2) et le volume (3D) comme le cube (3) du rayon (de dimension unitaire, 1D). Le bon sens et la géométrie sont cohérents avec cette valeur de $2 / 3$. Un système bio-physicochimique non sphérique perdrait plus vite la chaleur et consommerait davantage d'énergie pour un même résultat.

Les systèmes vivants sont des "entités thermodynamiques parcimonieuses, optimisées"!

Mais, graphiquement, $3 / 4$ et $2 / 3$ sont des pentes très proches. Et l'analyse statistique est trop imprécise pour faire la différence entre les 2 valeurs (figure 5).

Chez les oiseaux et les mammifères (niveau $\boldsymbol{i}+\mathbf{1}$ ), le métabolisme basal, en KiloJoule/kg/jour, est corrélé à la masse du corps (en $\mathrm{kg}$ ) par une loi de puissance $\mathbf{- 1 / 3}(-0,32)$. La dépense énergétique totale depuis la naissance jusqu'à la mort (LifeTime Energy Expenditure) en MégaJoule/kg et la masse du corps (en $\mathrm{kg}$ ) sont co-reliées par une loi de puissance -1/8 (-0,12). La durée maximale de survie (Maximum LifeSpan), durée de cette dépense énergétique vitale totale, est évidemment corrélée (négativement) au niveau minimal du métabolisme (métabolisme basal), mais par une loi de puissance $\mathbf{- 1 / 2}<<-\mathbf{5 / 8}(-0,51<<-0,61)$. Cette durée maximale est corrélée négativement ${ }^{18}$ au taux des peroxydations métaboliques selon une loi de puissance $\mathbf{- 2 / 5}(-0,40)$. Chez les organismes vertébrés (niveau $\boldsymbol{i}+\mathbf{1})$ il semble que la loi de puissance qui relie le métabolisme basal (mesuré par l'intensité respiratoire, en $\mathrm{ml} \mathrm{O} / \mathrm{h}$ ) à la masse du corps (en g) ait une pente plus élevée pour les endophysiotopes des organismes hétérothermes, ectothermes (amphibiens, poissons, reptiles), plus dépendants de leur écoexotope de survie, que pour les homéothermes, endothermes (mammifères, oiseaux), dont la capacité d'être accueilli de l'endophysiotope les rend plus indépendants de la capacité d'accueil de l'écoexotope de survie. Chez les oiseaux, le volume de la couvée (le nombre et la masse des oeufs) représente un double investissement en matière et en énergie, avec l'accumulation des réserves nutritives de l'oeuf, nécessaires à la croissance de l'embryon, et la production de chaleur nécessaire pour maintenir la température suffisante à la survie et au développement de l'embryon. Le volume de la couvée est proportionnel à la puissance $+\mathbf{1}$ de la masse du corps des parents qui couvent, que la couvaison soit paternelle, maternelle ou mixte. Survivre a un coût métabolique. Se survivre a un coût encore plus grand, c'est métaboliquement dispendieux chez toutes les espèces, mais "c'est un luxe nécessaire".

Chez les Angiospermes (niveau $\boldsymbol{i}+\mathbf{1}$ ), la teneur métabolique en azote d'une feuille (en $\mathrm{mg} / \mathrm{g}$ de feuille) est reliée à la surface foliaire (en $\mathrm{cm} 2 / \mathrm{g}$ de feuille) selon une loi de puissance $+\mathbf{2} / \mathbf{3}$. Pour la teneur en phosphore le coefficient de puissance est $+\mathbf{1}$. Dans le volume foliaire, en apparence à $\mathbf{2}$ dimensions, la teneur en azote rapportée à celle du phosphore suit aussi une loi de puissance $+\mathbf{2} / \mathbf{3}$.

Le rapport de la masse à la luminosité d'une étoile (niveau $i+4$, système solaire), d'une nébuleuse ou d'une galaxie (niveau $i+\boldsymbol{S}$ ), la mobilisation de matière et d'énergie, est reliée à l'éloignement dans l'espace par une loi également de puissance $\mathbf{2 / 3}$. Peut considérer que le volume correspondant est une sphère (figure 6) ? La masse est elle-même co-reliée à la luminosité par une loi de puissance $\mathbf{3 / 2}(3 / 2=1 / \mathbf{2} / \mathbf{3}) .^{19}$

\section{H. Les INTERACTIONS DIMENSIONNELLES inter-et intra-dimensions et systèmes ${ }^{20}: \underline{\text { SCALE power laws. }}$}

Chez les animaux (niveau $i+1$ ), le mouvement permet l'occupation de l'espace. Chez les mammifères, bipèdes ou quadrupèdes, qui courent, et les oiseaux, qui volent, la vitesse maximale (le déplacement dans l'espacetemps, en $\mathrm{km} / \mathrm{h}$ ) est reliée à la masse corporelle (en $\mathrm{kg}$ ) par une loi de puissance $+\mathbf{1 / 6}$.

statistical parameter which characterizes the robustness of a population, and describes the uncertainty in the age of the mother of a randomly chosen newborn. Entropy has a macroscopic description: it is linearly related to the logarithm of the variables body size, metabolic rate and life span. Entropy could characterize Darwinian fitness, the efficiency with which a population acquires and converts resources into viable offspring. Accordingly, entropy predicts the outcome of natural selection in populations subject to different classes of ecological constraints. This predictive property, when integrated with the macroscopic representation of entropy, is the basis for enormous differences in morphometric and life-history parameters across species [60].

18 L'oxygène activé, les radicaux libres, les peroxydes, tous les agents oxydant ou altérant la matière organique, en particulier les structures membranaires qui séparent les compartiments intra-cellulaires (niveau $i-1$ ), sont délétères et réduisent la durée de survie des structures.

$19 \mathbf{3} / \mathbf{2}=1 /(\mathbf{2} / \mathbf{3}), \mathbf{2} / \mathbf{3}=1 /(\mathbf{3} / \mathbf{2})$; suivant la structure du graphe de lecture de la relation, suivant la variable prise pour cause, on trouve $2 / 3$ ou $3 / 2, !$ Et la cause peut être l'effet et l'effet peut être la cause (Loi systémique constructale), tout est relatif à l'espace-temps de référence, ce qui dépend du "point de vue" de l'observateur...

20 Examples of power laws exponents : $\mathbf{1 / 6}=0.166, \mathbf{1} / \mathbf{5}=0.200, \mathbf{1} / \mathbf{4}=0.250, \mathbf{1 / 3}=0.333, \mathbf{2} / \mathbf{5}=0.400, \mathbf{1} / \mathbf{2}=0.500, \mathbf{4} / \mathbf{7}=0.571, \mathbf{3} / \mathbf{5}=$ $0.600, \mathbf{2} / \mathbf{3}=0.666$ (sphere surface/volume ratio), $\mathbf{3} / \mathbf{4}=0.750, \mathbf{4 / 5}=0.800, \mathbf{5} / \mathbf{6}=0.833, \mathbf{5} / \mathbf{5}=1.000, \mathbf{6} / \mathbf{5}=1.200, \mathbf{5} / \mathbf{4}=1.250, \mathbf{4} / \mathbf{3}=1.333$, $\mathbf{7 / 5}=1.400, \mathbf{3} / \mathbf{2}=1.500, \mathbf{8} / \mathbf{5}=1.600, \mathbf{5} / \mathbf{3}=1.666, \mathbf{7 / 4}=1.750(\mathrm{DLA}), \mathbf{9} / \mathbf{5}=1.800, \mathbf{1 0} / \mathbf{5}=2.000, \mathbf{1 1} / \mathbf{5}=2.200, \mathbf{7 / 3}=2.333$ 
La "durée fonctionnelle" (en années humaines) d'un cycle de mobilisation de la matière et de l'énergie d'un agro-système (anthropo-système agricole) ou d'un éco-système naturel (biotope sauvage, sans l'homme) [32] est proportionnelle à la puissance $+\mathbf{1}$ de sa superficie (en $\mathrm{km} 2)$.

Dans tout écosystème (niveau $\boldsymbol{i}+\boldsymbol{2}$ ), la "durée d'atteinte du climax" (en années, ou en saisons d'activité) est proportionnelle à la superficie (en $\mathrm{km} 2$ ) selon une puissance qui dépend des acteurs et de leur lieu d'intégration dans la chaîne alimentaire : $+\mathbf{3} / \mathbf{7}$ pour les végétaux producteurs (de matière et d'énergie), $+\mathbf{7 / 7}$ pour les animaux herbivores, consommateurs. C'est la même valeur (7/7) pour tout phénomène de perturbation de l'écosystème. Et l'arrivée d'un ravageur, d'un prédateur ou d'un parasite, est toujours une perturbation de l'écosystème pour ses proies ou ses hôtes!

Les interactions entre l'espace et le temps permettent la mise en place d'une organisation temporelle dans un espace donné ou/et d'une organisation spatiale à un moment donné et pour un temps donné (tableau 1). Cette mise en place se fait par percolation (figure 1.-8). ${ }^{21}$

"Un temps pour chacun, chacun dans son temps. Une place pour chacun, chacun à sa place."

La performance, en pourcentage du maximum (atteint avec le passage à l'état adulte reproducteur), diminue inéluctablement, de façon constante (cinétique du premier ordre), avec l'avancée en âge. L'allongement de la durée de vie n'y change rien. Chez l'homme et les mammifères, prenons comme "unité de référence" (1X) de cette diminution le paramètre vital le moins affecté par le "vieillissement", la vitesse de conduction de l'influx nerveux. Elle ne diminue que de $10 \%$ dans les 60 années qui suivent l'âge du maximum. La performance cardiaque (la fréquence maximale du rythme cardiaque) diminue $3 \mathrm{X}$ plus vite. Le débit sanguin rénal et la filtration glomérulaire diminuent encore plus vite. La capacité de travail maximale (maximum work rate), mesurée par la consommation d'oxygène (oxygen uptake), diminue 7X plus vite. Et chez les femelles, le potentiel de fertilité, qui est la fonction qui disparaît le plus rapidement, diminue $30 \mathrm{X}$ plus vite. "Se survivre a un coût énorme, qui ne peut être payé qu'un temps limité" (entre la puberté et la ménopause chez la femme).

$\mathrm{Au}$ voisinage d'un trou noir galactique (niveau téloscopique $i+\varsigma$ ), le décalage temporel (le temps de réponse, en $\mathrm{ms}$ ) de la rotation du jet de production de rayons X (la mobilisation de la matière et de l'énergie, le métabolisme !) est lié à l'énergie des photons (en $\mathrm{keV}$ ) par une loi de puissance +1. À l'opposé dimensionnel, au niveau des particules sub-atomiques (niveau picoscopique $\boldsymbol{i}-\boldsymbol{\sigma}$ ) d'un courant électrique (flux de matière et d'énergie), la résistance (le frein au mouvement) et la force électromotrice sont liées par une loi de puissance -1.

Les lois de puissance sont présentes, en tout temps et en tout lieu, dans tous les systèmes organisés hiérarchisés, quel que soit le niveau d'organisation.

\section{Les INTERACTIONS entre capacités, entre acteurs et au sein d'un acteur : INTEGRATION power laws.}

Le nombre de types de pièces (aspect qualitatif) et le nombre des pièces (aspect quantitatif) d'un jeu de lego sont liés par une loi de puissance en $\mathbf{+} \mathbf{0 , 7 1}$ (entre $\mathbf{2 / 3}=0,66$ et $\mathbf{3 / 4}=0,75$ ). Une loi de puissance, graphiquement $\log (y)=K \log (x)$, est aussi une fonction hyperbolique telle que $y x=k .^{22}$ Il y a une limite à la fois au nombre de types de pièces possibles et au nombre possible de pièces, (qualité) $\mathbf{x}$ (quantité) = constante. Ces limites sont liées au volume d'espace occupé localement par chaque acteur et au volume global disponible pour tous. La croissance en nombre est inversement proportionnelle à la croissance en taille (corrélation négative : loi de puissance -1) : "peu de beaucoup" ou "beaucoup de peu“, $\mathrm{yx}=\mathrm{k}$ ! [31,32]

Les capacités d'intégration, de réaction aux stimulations et d'organisation spatiale et temporelle (figures 2 \& 3) sont liées par des lois de puissance. La propagation des potentiels d'action, sommables dans l'espace et le temps, entre les neurones du cerveau des mammifères, in vitro, et in vivo, avec ou sans anesthésie, à l'état de veille ou de sommeil, obéit à des lois de puissance, quelle que soit la densité des neurones et la nature du signal déclenchant.

21 La percolation est initialement le phénomène de passage de l'eau (d'un fluide) à travers la poudre (les grains) du café (d'un sol). On a, entre les grains (un type d'acteurs), des pores (un autre type d'acteurs ?) reliés entre eux (les interactions) qui forment des "amas" (des structures) où l'eau peut circuler. Pour une valeur précise du nombre de pores (le seuil de percolation) il existe un chemin, "un lien" percolant, qui relie les frontières supérieure (entrée dans le système) et inférieure (de sortie) du Tout; le fluide passe, percole. Les pores, acteurs locaux, ont acquis la propriété collective, globale, émergente, de laisser passer le fluide au travers des grains. On parle par extension de percolation quand des acteurs, en nombre suffisant, indépendants localement, coopèrent globalement pour former un tout unique, solidaire, qui acquiert des propriétés émergentes que ne possédaient pas les acteurs locaux "libres".

Quantities describing network structure such as modularity, degree-degree correlation, shortest pathway topology and signal load can be used to describe yeast protein networks [51].

22 Si yx $=\mathbf{k}, \log (\mathrm{xy})=\log (\mathrm{k}), \log (\mathrm{x})+\log (\mathrm{y})=\mathbf{K}, \mathbf{Y}+\mathbf{X}=\mathbf{K}, \mathbf{s i} \log (\mathrm{y})=\mathbf{k} \cdot \log (\mathbf{x}), \mathbf{y}=\mathbf{x}^{\mathrm{k}}$ 
La capacité de réaction à des stimulations chez les vertébrés aériens peut être mesurée par la compliance pulmonaire ${ }^{23}$ (en $\mathrm{ml} / \mathrm{cm} \mathrm{H} 20$ ). Elle est reliée à la masse corporelle par une loi de puissance $+\mathbf{1}$ (corrélation positive). Chez les mammifères, la surface alvéolaire (en $\mathrm{m} 2$ ) et la ventilation pulmonaire ou/et la consommation d'oxygène (en $\mathrm{ml} / \mathrm{min}$ ) sont corrélées suivant une loi de puissance $+\mathbf{1}$. Chez le criquet pèlerin [52], la respiration mesurée par le volume des sacs aériens (en $\mathrm{ml}$ ), rapportée au poids frais (en g), suit une loi de puissance de coefficient $\mathbf{+ 1 , 7 2}$ en expiration (déflation) et $+\mathbf{1}, \mathbf{3 7}$ en inspiration (inflation), soit en moyenne $\mathbf{+ 3 / 2}(=1,50)$. La respiration (de l'ENDO) mesurée par la ventilation pulmonaire (en $\mathrm{ml} / \mathrm{min}$ ), rapportée au poids frais (en $\mathrm{g}$ ), suit une loi de puissance en $\mathbf{+ 1 , 2 6}$ quand la teneur en $\mathrm{O} 2$ (de l'EXO) est de $21 \%$, et en $+\mathbf{1 , 3 6}$ quand cette teneur est de $5 \%$. La capacité d'être accueilli de l'ENDO dépend non seulement de la capacité d'accueil de l'EXO mais aussi de leurs interactions.

Tout se passe comme si, quel que soit le comportement de survie, si on étudie les paramètres représentatifs de l'invariance de jauge (figure 2.-1) on peut mettre en évidence une loi de puissance les reliant, par exemple entre la fréquence d'ébrouement d'un animal (mouvement) et sa masse (croissance)... (ici -0,22 [44])!

\section{LE TABLEAU DE CLASSIFICATION PÉRIODIQUE DES SYSTĖMES VIVANTS.}

Un comportement de groupe et la mise en place d'une organisation sont nécessaires mais ils ne suffisent pas à définir un organisme. Tout organisme est défini par une interface de séparation qui isole l'intérieur (l'endophysiotope) de l'organisme du milieu extérieur de survie (l'écoexotope), il est formé de parties différentes qui constituent, ensemble, un Tout indissociable et qui sont spécialisées dans des capacités fonctionnelles différentes (figure 1). Des propriétés nouvelles apparaissent dans le tout (niveau $i$ ). Le tout est plus que la somme de ses parties (niveaux $i-1$, i2 ...) : il y a mise en place d'un niveau supérieur d'organisation, SURMESURE des niveaux précédents, et SUR MESURE... avec des propriétés nouvelles "émergentes" spécialisées. Mais, simultanément des capacités du niveau d'organisation inférieur sont perdues. Le tout est moins que la somme de ses parties. Tout niveau d'organisation est donc défini par son échelle d'emboîtements (figure 1) [33, 36].

\section{$J$. Thermodynamique, entropie, et organisation de l'ESPACE-TEMPS-ACTIONS}

Les lois de la thermodynamique ${ }^{24}$ définissent la température, l'énergie et l'entropie, grandeurs physiques qui permettent de caractériser les systèmes dits thermodynamiques. Elles décrivent comment ces quantités varient et quels changements sont possibles. La première loi dit que l'énergie (sous forme de matière, de chaleur, de travail) d'un système clos est invariablement conservée. Elle ne peut changer qu'en système ouvert, par des transferts, d'origine externe ou interne. Rien ne dit que l'Univers soit un système fermé. Au contraire, l'homothétie fractale mise en évidence avec la loi de croissance de puissance $+3 / 2$ (figure 3) indique que, comme tous les sous-systèmes qui l'habitent, l'endophysiotope de Univers "habite" un écoexotope de survie qu'il peut partager avec d'autres Univers. La seconde loi indique que l'entropie d'un système clos qui n'est pas en équilibre thermique ne peut qu'augmenter, l'état d'équilibre physique étant celui d'entropie maximale. À partir d'un certain âge, l'Univers atteindrait un état où l'énergie serait uniformément distribuée, sans différences thermiques exploitables pour un travail. Dès l'instant où il n'existerait plus de différences thermiques, à l'équilibre thermique (au maximum d'entropie), l'absence d'énergie disponible ne permettrait plus d'entretenir les processus de survie consommateurs d'énergie. Le mouvement d'expansion de l'Univers convertissant l'énergie disponible en travail et en chaleur, l'uniformité thermique augmente globalement. À l'inverse, dans les systèmes vivants, l'ordre s'accroit, l'entropie diminue. Localement, l'hétérogénéité thermique s'accroît. Les systèmes vivants sont des systèmes ouverts, utilisant parcimonieusement la matière et l'énergie afin d'obtenir un maximum de production d'organisation pour un minimum de production d'entropie. [33, 34]

L'entropie est une mesure d'un manque d'information sur le niveau d'organisation microscopique dans un système macroscopique. Pour deux états macroscopiques d'un même système il existe entre eux une différence d'état informationnel, une différence d'entropie. Cette différence indique quelle quantité d'information supplémentaire est nécessaire pour passer de l'un à l'autre. Tout système naturel (figure 1) de niveau d'organisation $\boldsymbol{i}$ (figure 2) est un assemblage, par juxtaposition et emboîtements, de sous-systèmes (acteurs, processus) de niveaux d'organisation $i-1$, $i-2, \ldots, i-n$ qui ne sont pas exactement connaissables et prédictibles, même si on en connaît parfaitement les conditions initiales et les lois qui les gouvernent (figure 3). C'est la raison pour laquelle l'entropie augmente au cours des processus naturels, par perte d'énergie (dissipation) et consommation d'énergie pour un travail (mouvement).

La mort de l'Univers comme celle de tout système vivant est une mort de repos thermique ${ }^{25}$ inéluctable.

\footnotetext{
23 aptitude d'une cavité organique à changer de volume sous l'effet d'une variation de pression

24 http://en.wikipedia.org/wiki/Laws of thermodynamics

25 http://en.wikipedia.org/wiki/Heat death of the universe
} 
Survivre c'est manger et ne pas être mangé. La durée de survie est limitée !

Tôt ou tard il est impossible de ne pas être mangé.

\section{K. Peu importe les lois de la thermodynamique... Elles pourraient être autres!}

Personne n'a jamais vu un électron! Dans des détecteurs appropriés, nous ne voyons que des traces, laissées par sa présence, qui sont analysées par l'utilisation de modèles. Nous ne percevons pas sa forme, mais l'électron est sphérique car la distribution statistique de sa charge est dans un volume parfaitement sphérique [66].

Sans la liaison chimique nous n'existerions pas. Par leur liaison les atomes solitaires deviennent solidaires. Par leur union électrostatique ils perdent leur individualité en formant un nouveau tout [62]. De la liaison chimique découlent les propriétés structurales et fonctionnelles de la matière, la manière dont les mêmes atomes (niveau $i-4$ ) sont liés explique les différences moléculaires (niveau adjacent supérieur $\boldsymbol{i}-\boldsymbol{B}$ ). Sans le phénomène de mise en place de ARMSADAs nous n'existerions pas! Dans le tableau périodique de Mendeleev les atomes d'une même colonne forment le même nombre de liaisons. Cette propriété est conservée dans la molécule (semi-autonomie intramoléculaire). En se liant (juxtaposition), les atomes mettent en commun, donnent ou reçoivent des électrons. Ils sont à la fois plus et moins que ce qu'ils étaient avant, et, leur tout est à la fois plus et moins que la somme de ces parties [29]. Dans le tableau périodique du vivant (figure 2), de la même façon les systèmes d'une même ligne sont équivalents et les systèmes d'une même colonne partagent des propriétés qui sont conservées tout au long de la colonne. Qu'importe les lois thermodynamiques, elles pourraient être autres! C'est la "visualisation" d'un algorithme [41] : un processus itératif, une succession d'étapes, une liste ordonnée de règles et de consignes (l'invariance de jauge) à respecter en vue de parvenir à un résultat (la mise en place d'une ARMSADA), avec une condition de croissance (figure 3) et de finitude ("survivre c'est manger et ne pas être mangé, tôt ou tard il est impossible de ne pas être mangé") et une contrainte (le fonctionnement en flux tendus constants) (figure 4).

Dans le monde quantique, de l'échelle de Planck à l'échelle des quarks (figure 3a), en-deçà de la limite du "connu“, si on remplace (symétrie miroir) le temps et l'espace l'un par l'autre (cases grises, flèche orange), on obtient un meilleur ajustement linéaire de pente $+3 / 2$ (droite en gris).

Tout se passe comme si le temps crée l'espace et l'espace crée le temps. [27, 28, 29]

\section{L'homothétie fractale du vivant: $\mathbf{V}_{\mathrm{A}}=\mathbf{C} \cdot \mathrm{t}_{\mathrm{g}}{ }^{3 / 2}$, "Into and Out Matryoshka Climbing up" (figure 3b.-4-)}

Quand on passe du Tout aux parties ( figure $3 b$, de $\boldsymbol{n}$ à $\boldsymbol{n}-1$, flèches noires : niveaux PICOscopique, MACROscopique ou TÉLOscopique, par exemple) et inversement, quand on passe des parties au Tout, ou "au-dessus du Tout" à "ce qui contient le Tout" (figure $3 b$, de $n$ à $n+1$, flèche jaune), on observe toujours la même loi de puissance 3/2. L'Uni-vers lui-même est donc inclus dans un Multi-vers, qui le contient, emboîté, et juxtaposé avec d'autres Univers (de même nature et de natures différentes), de la même façon que notre Univers (niveau $\boldsymbol{i}+\mathbf{7}$ ) inclut, par emboîtement et juxtaposition, les sous-systèmes dont notre forme de vie est une des sous-sous-...-sous-parties (niveau $\boldsymbol{i}+1$ ) (par rapport au niveau d'organisation, arbitraire, de référence, le niveau cellulaire, niveau $\boldsymbol{i}$, figure 2).

Quels que soient le niveau d'organisation et le degré d'intégration d'un système vivant, le flux des échanges, le "débit" rapporté à la surface d'échange ( $\mathbf{D}^{2}$ à $\mathbf{t}_{\mathbf{g}}$ ), à l'interface entre écoexotope et endophysiotope, est globalement constant ( $\mathbf{k}^{\prime}$, figure 4-3). Ce qui conforte mon hypothèse relative à l'origine de la cellule [22, 24].

\section{Une LOI systémique Constructale, De PUISSANCE 3/2. A GENERALIZED KEPLER'S THIRD LAW LIKE LAW}

\section{Le mouvement brownien, loi de puissance 3/2, processus fonctionnel, constitutif et cause de notre Univers ?}

Un espace-temps siège de fluctuations et singularités aléatoires, en apparence désordonnées, dans un type d'entrelacement chaotique ou en mouvement dit "brownien", peut être ressenti comme "complexe". Un système physique dynamique avec 3 états, de Pression, Température et Volume, reliés par un système mathématique de 3 équations différentielles, est un système complexe d'effets browniens d'agitation thermique. Le mouvement brownien d'une particule suspendue dans un liquide est complètement irrégulier, mais s'il y a beaucoup de particules similaires, elles donneront naissance, par leurs mouvements irréguliers locaux, au phénomène régulier global de la diffusion. Le mécanisme d'agrégation limitée par diffusion (DLA), de particules en mouvement aléatoire, est régi par une loi de puissance proche de $\mathbf{3} / \mathbf{2}$.

La fractalité du vivant, loi de puissance $3 / 2, V_{\mathrm{A}}=\mathrm{C} . \mathrm{t}_{\mathrm{g}}{ }^{3 / 2}$, reflète-t-elle le mouvement brownien sous-jacent ? $3 / 2$ est aussi l'exposant de l'augmentation relative du volume rapporté à la surface d'une sphère (figure 6). 


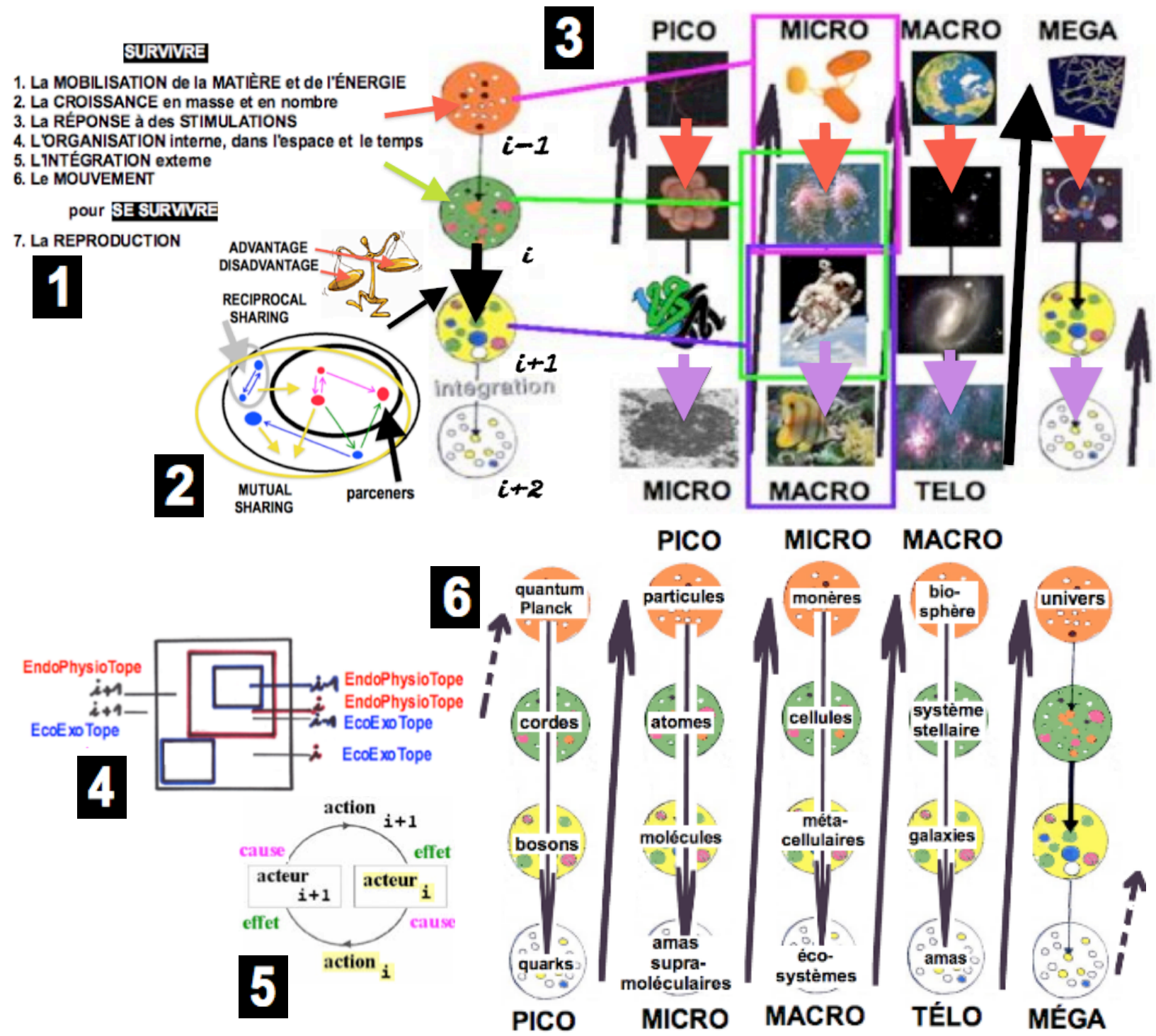

Figure 2. The periodic classification chart of Living Systems.

Le tableau -3- est construit en prenant en compte, simultanément, le fait que tout système vivant, quel que soit son niveau d'organisation -6- possède 7 caractéristiques mutuellement nécessaires et suffisantes (invariance de jauge) -1-, et le fait que tout nouveau niveau d'organisation -6- résulte de la mise en place d'une ARMSADA -2-. Chaque case du tableau -3- correspond à un niveau d'organisation qui emboîte les niveaux précédents et qui est emboîté dans les niveaux suivants (poupées gigognes) -4- (figure 1.3). De fait tout niveau d'organisation est un acteur local d'un niveau d'organisation supérieur adjacent et un Tout qui contient des acteurs de niveaux d'organisation inférieurs -5-. Du quantum de Planck à l'Univers entier, les flèches noires des tableaux (-3- et -6-) indiquent la "montée de complexité", de l'infra-microscopique (PICO) au microscopique (MICRO) puis au macroscopique (MACRO) et au supra-macroscopique (TÉLO, MÉGA). Tout niveau d'organisation (tableau -3- : flèche orange niveau $\boldsymbol{i}-1$ en orange, flèche verte niveau $\boldsymbol{i}$ en vert...) possède les 7 caractéristiques $-1-$. Le passage d'un niveau au suivant (schéma -2- et tableau -3-) : de $\boldsymbol{i}$ à $\boldsymbol{i}+\mathbf{1}$ flèche noire (-2-), de $i-1$ à $\boldsymbol{i}$ (du niveau des Monères au niveau unicellulaire $-\mathbf{3 -}$ ) flèche et cadre rouges, de $i$ à $i+1$ (du niveau cellulaire au niveau méta-cellulaire) cadre vert, de $i+1$ à $i+2$ (du niveau des espèces métacellulaires aux écosystèmes terrestres) flèche et cadre violets, s'effectue par la mise en place d'une ARMSADA -2-. Toutes les cases d'une même colonne (-3- et -6-) regroupent des systèmes dont les acteurs possèdent le même type d'interface entre endophysiotope et écoexotope. Les flèches de même couleur (rouge, en début de colonne par exemple -3-) indiquent des processus de passage équivalents. Les cases, dans des colonnes différentes, mais à la même place correspondent à des systèmes "équivalents". 

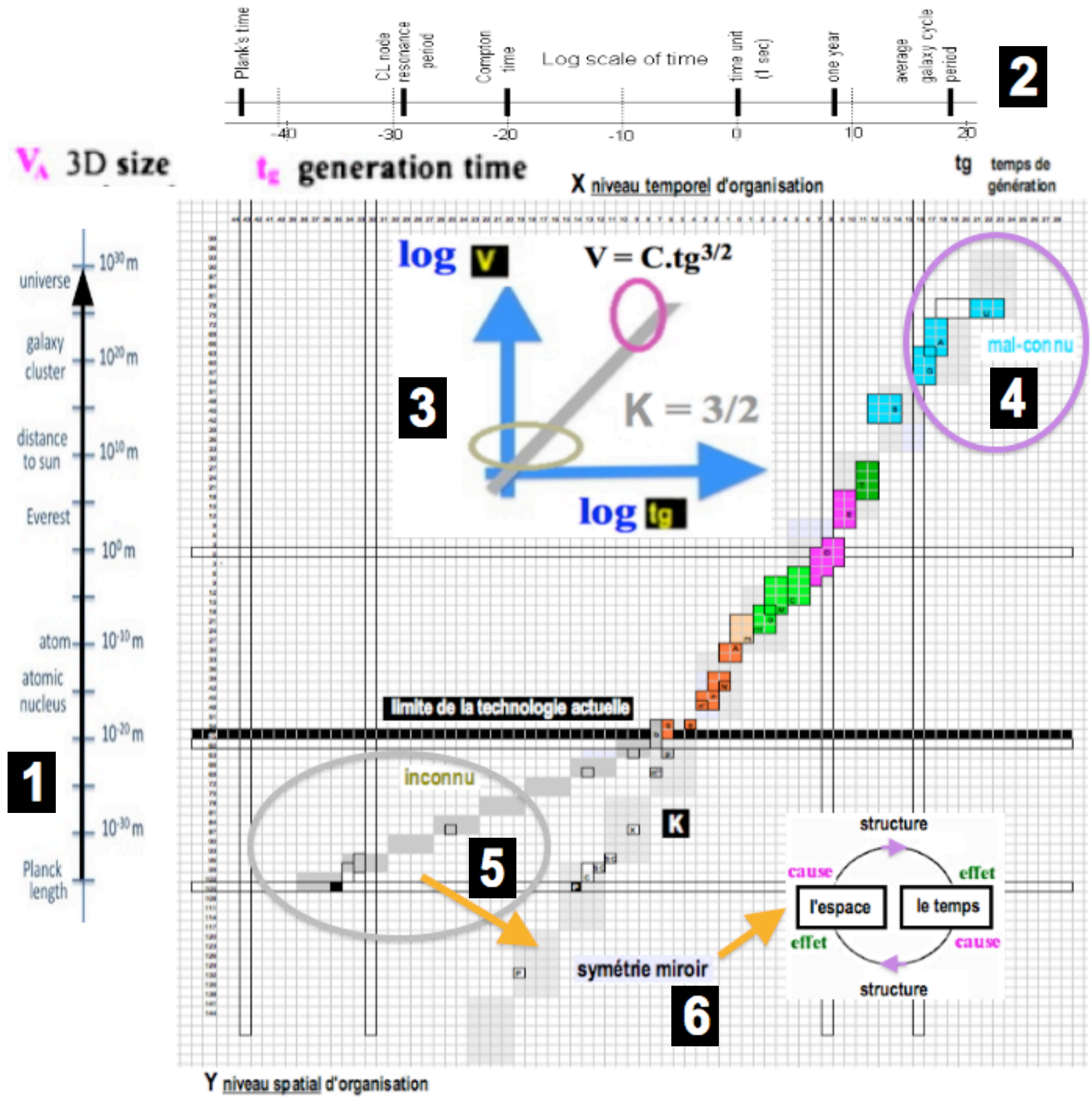

Figure 3. A Kepler's third Law like relation.

Figure 3a. Ordres de grandeur de quelques dimensions, spatiales (1D) : diamètre apparent de l'Univers 10+26 m, de la terre 10+7 m, d'une cellule 10-5 m, de l'atome 10-10 m, du noyau 10-15 m, du proton ou neutron 10-16 m, de l'électron 10-17 m, des quarks 10-18 m, d'une corde 10-34 m, longueur de Planck 1,6 x 10-35 m, taille initiale de l'Univers : 10-35 m à 10-43 s et temporelles : âge de l'Univers plus de 10+18 s, temps de génération d'une cellule 10+4 s, durée de vie d'un boson ou d'un quark libre 10-24 s, temps de Planck 5,4 10-43 s (pour plus de détails http://www.armsada.eu/pb/bernardins/phylotagmotaphologie.pdf).

En ordonnées (Y niveau spatial d'organisation, -1- : échelle 1D en puissances de 10 ; échelle du volume 3D, en puissances de 3Y) : le volume à l'état adulte VA (du système supposé sphérique).-2- En abscisses ( $\mathbf{X}$ niveau temporel d'organisation, échelle indiquée en puissances de 10) : le temps de génération tg, durée d'atteinte de l'état adulte (durée de la phase de croissance). $\mathbf{- 3 -} \mathrm{K}=\mathbf{+ 3 / 2}$ -4- domaine du monde cosmique, mal connu et modélisé, -5- domaine du monde quantique, inconnu, modélisé et simulé, -6- symétrie miroir (échange des valeurs de l'espace et du temps). 


\section{4}

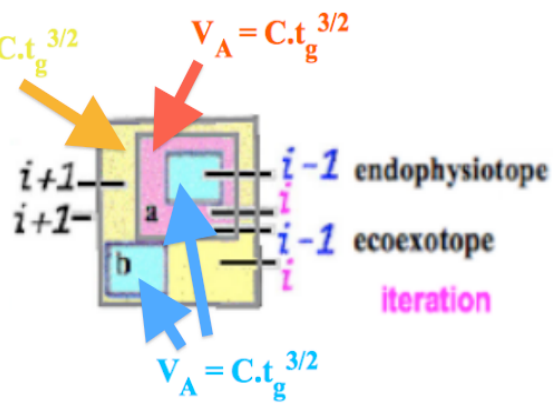

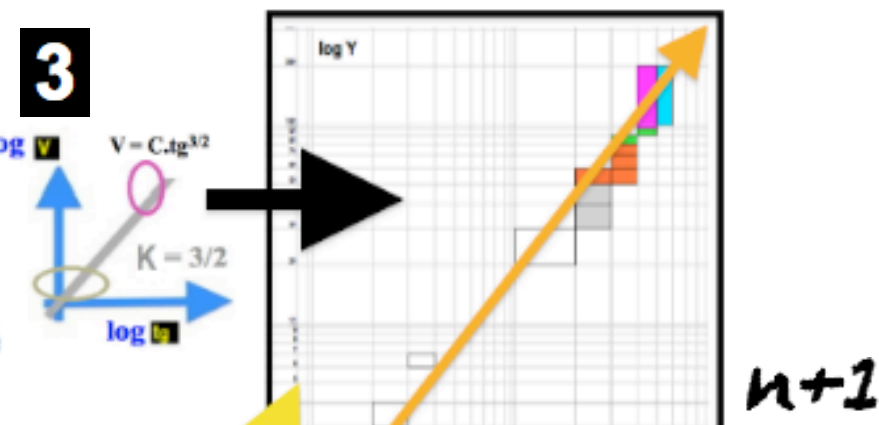

$\log x$

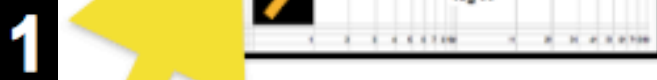

1
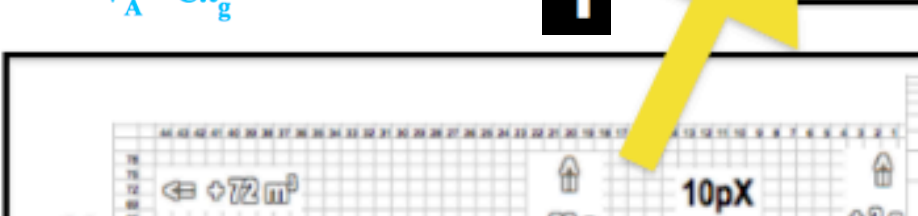

$+20$ क †凬

$10 \mathrm{pX}$

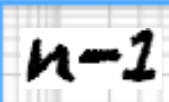

- 288

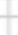

$+10$

争

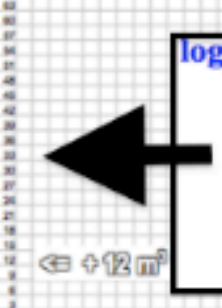

$V_{A}$

$-10$

. $6.8 \pi^{2}$

g V $\quad \mathrm{V}=\mathrm{C.tg}^{\mathrm{V} 2}$
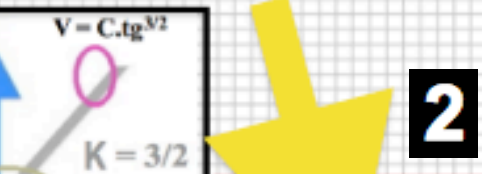

$\mathrm{K}=3 / 2$

$\log$ a

MACRO

3

$10 p 3 Y$
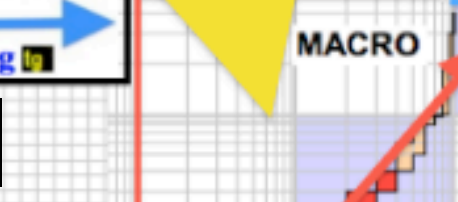

$-20$

$-30$

.

$\Leftrightarrow \circ 00 \mathrm{~min}^{2}$

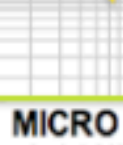

MICRO
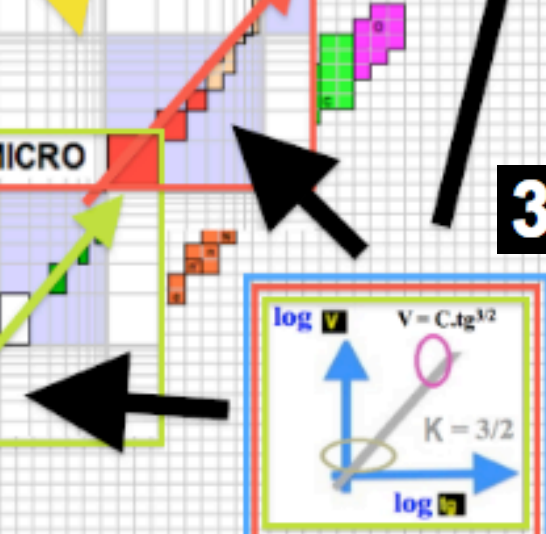

II
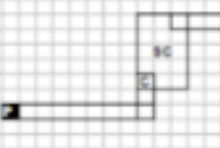

$-30$

$-20$

$-10$

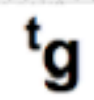

Figure 3b. Loi d'homothétie fractale inter- et intra- niveaux d'organisation : $\mathbf{V}_{\mathbf{A}}{ }^{2}=\mathbf{C . t}_{\mathbf{g}}{ }^{3}$

À l'échelle de l'Univers entier (cadre noir, niveau $\boldsymbol{n}$ ), comme à l'échelle des systèmes parties de notre Univers (cadres et flèches, vert, rouge ou bleu, niveaux adjacents inférieurs $\boldsymbol{n}-\mathbf{1}$ ), comme à une échelle au-delà de notre Univers (niveau adjacent supérieur $\mathbf{n + 1}$ ), aussi bien quand on descend en organisation (flèche orange -2-) que quand on monte en organisation (flèche orange -1-), quel que soit le degré d'emboîtement et de juxtaposition (-4- flèches), quelle que soit la place de l'acteur, local ou global [29], la même loi de puissance $\mathbf{+ 3 / 2}$ ( $-\mathbf{3}$ - flèches noires) relie toujours le temps de génération tg (durée d'acquisition de la capacité reproductrice) et le volume au moment de l'acquisition de l'état adulte VA, l'état de croissance en volume (figure 4.-2). 


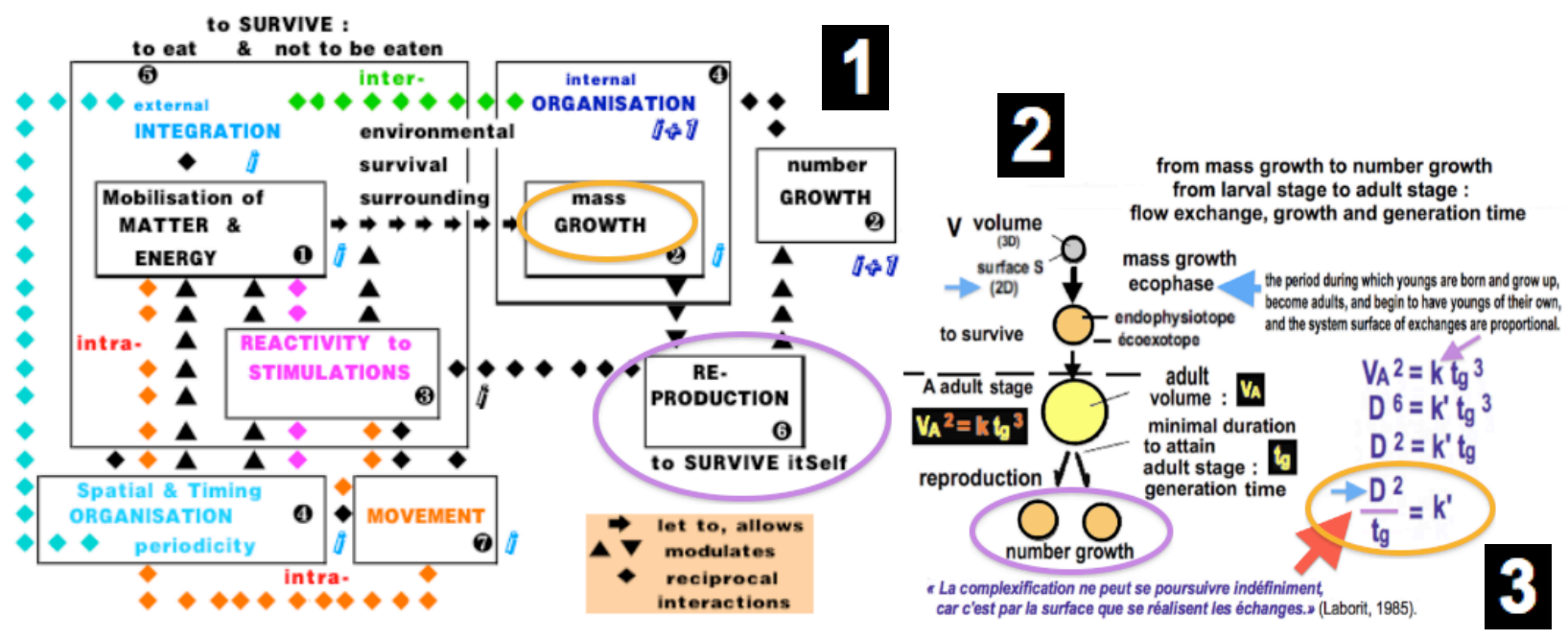

Figure 4. Mobilisation de la matière et de l'énergie à flux constant.

Matter and energy mobilization : exchanges at constant flows.

-1- gauge invariance : To survive with mass growth is the prerequisite for the life form to itself survive with number growth. $-2-V_{A}=C_{.} t_{g}{ }^{3 / 2}: 3 / 2$ is just the true exponent that describes "the ratio of surface area to volume in a sphere".

-3- Just-in-time exchanges : D space dimension, the flow of exchanges reported to the surface of exchange $\mathbf{D}^{2}$ is a constant $\mathbf{k}^{\prime}$.
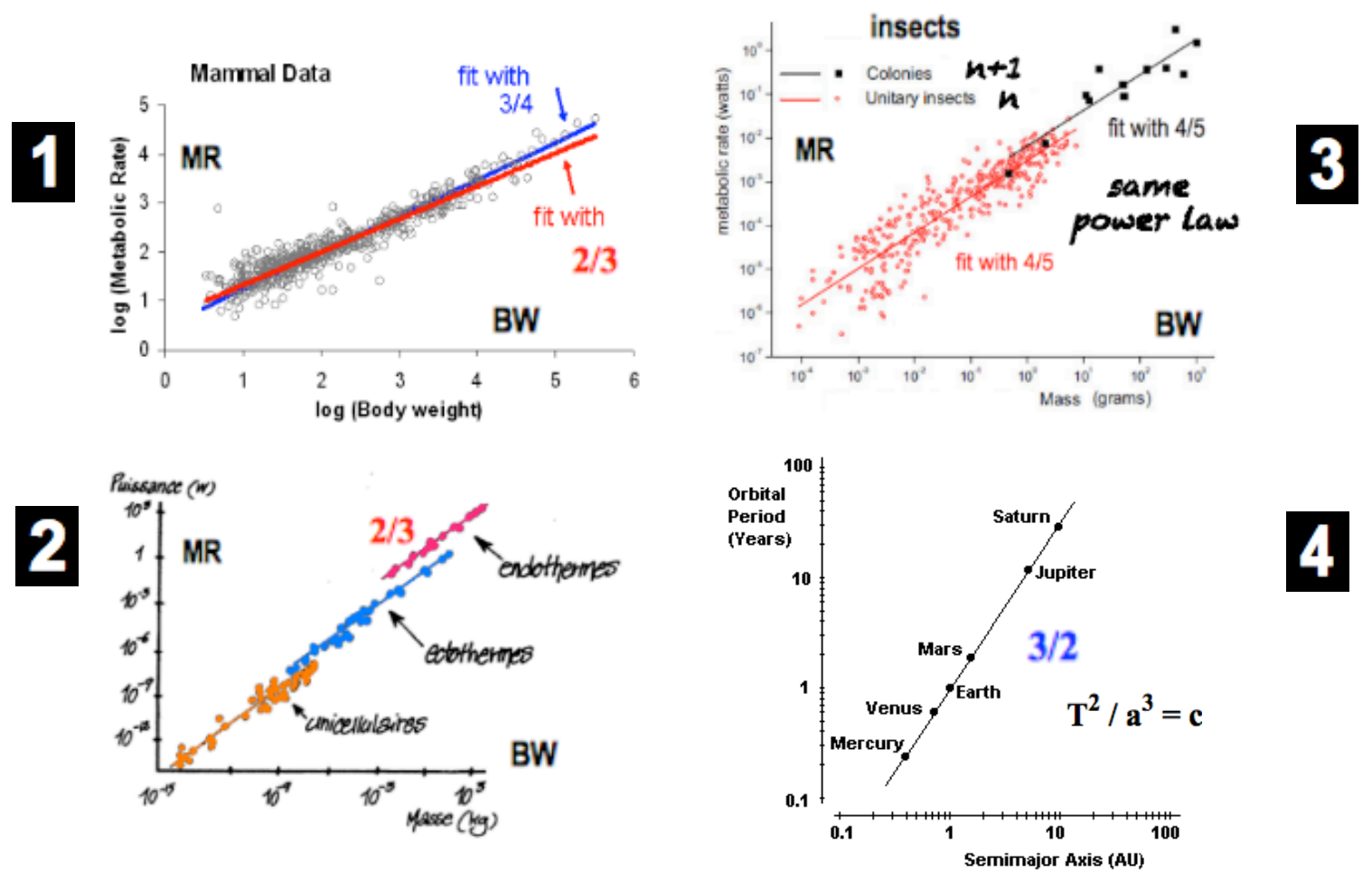

Figure 5. Co-relations power laws between Living Systems "functional dimensions".

-1- \& -2- metabolic power law (MR metabolic rate, BW body mass) : whatever the units and living forms, exponent fits with $2 / 3$. -3- except for insects... 4/5 ?, data are more heterogenous, but with the same power law for individual or colonial insects.

-4-Kepler's $3^{\text {rd }}$ law of the solar system ( $i+4$ organization level) : a 3/2 exponent power law (cube of space a=c.square of time T) 


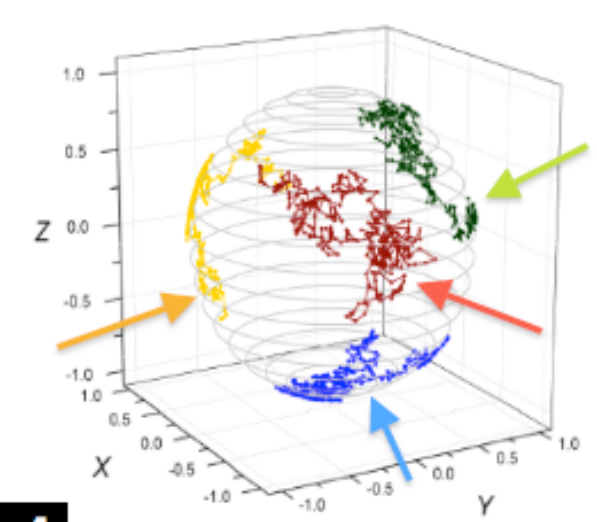

trajectories of particles

projection onto the surface of a unit sphere

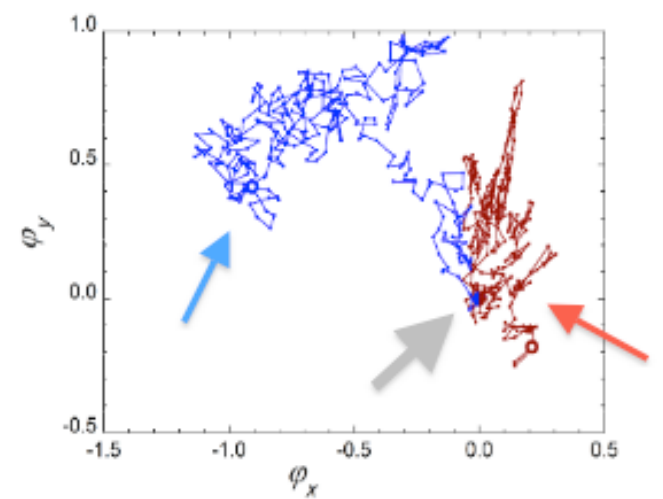

projection of two trajectories through rotation space

simulated Brownian tetrahedral cluster

Colors represent different particles
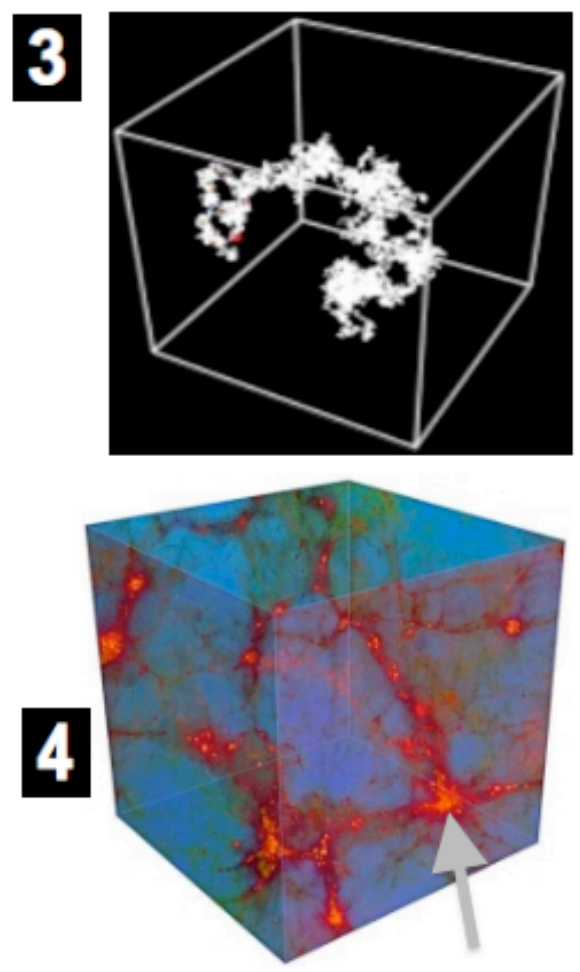

Colors correspond to the same particles

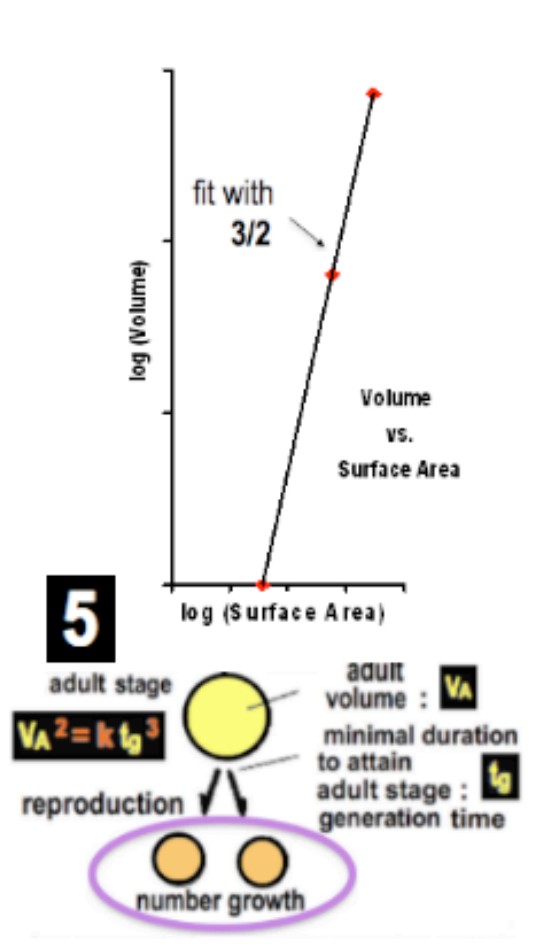

$\mathrm{X} 2$
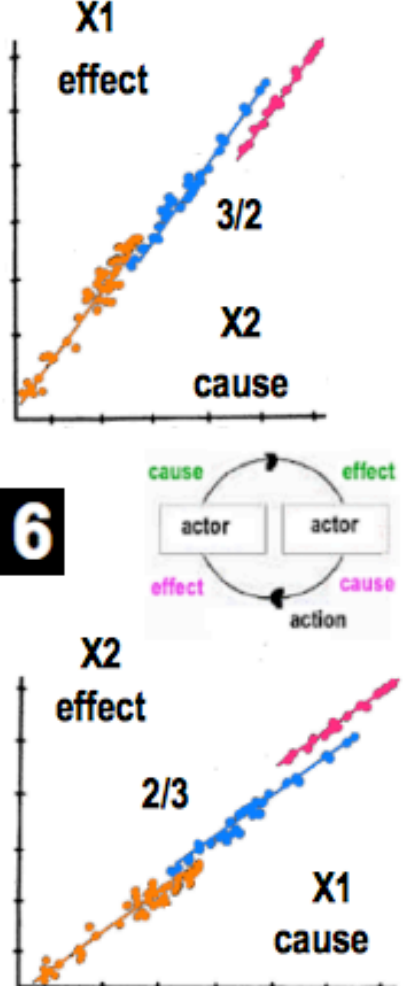

Figure 6. Brownian movement and structures, $3 / 2$ or 2/3 power laws of co-relation.

-1-3D projection of the Brownian movement of free particles ( $\boldsymbol{i}-\boldsymbol{S}$ level) onto a sphere (a different color for each particle),

-2- the same onto a surface (2D projection), particles trajectories may overlap (grey arrow).

-3- 3D projection of the simulation of the Universe Brownian structure $(i+7$ level),

-4- a virtual spongy structure of the Universe matter (grey arrow) with 10,000 galaxies in a 260 millions light years cube.

-5- 3/2 is just the true exponent that describes "the ratio of volume to surface area of a sphere",

-6- the exponent, $\mathbf{2 / 3}$ or $\mathbf{3 / 2}$, is depending on which of the measures, X1 or X2, we are mentioning as cause or effect, particularly when $\mathbf{X 1 . X 2}=\mathbf{k} \ldots$, so $2 / 3$ or $3 / 2$ ? ... $2 / 3=\mathbf{1} /(\mathbf{3} / \mathbf{2})$. 
La valeur de l'exposant observé, $2 / \mathbf{3}$ ou $\mathbf{3} / \mathbf{2}$, puisque $\mathbf{2 / 3}=\mathbf{1} /(\mathbf{3} / \mathbf{2})$, dépend d'abord des paramètres mesurés, et considérés a priori comme cause ou effet (X1 et X2, figure 6), il s'agit d'une co-relation, non d'une corrélation. La valeur de la masse peut être la cause de la durée de la phase de croissance, il faut atteindre une masse minimale pour acquérir la capacité de se survivre. La masse dépend de la capacité d'accueil de l'écoexotope de survie, mais elle résulte aussi de la capacité d'être accueilli qui détermine l'utilisation de cette capacité d'accueil. La valeur de la masse est aussi l'effet de cette interaction ente capacité d'accueil et capacité d'être accueilli. La durée de la phase de croissance est alors cause de l'accumulation de la masse. Le volume d'un organisme est lié à sa masse et à sa forme; il est limité à la fois par la durée de la phase de croissance et les contraintes de la mise en place de son organisation, spatiale et temporelle. Le volume peut être l'effet (X2) ou la cause (X1) de la durée de la phase de croissance (X1 ou $\mathrm{X} 2$ ), selon le point de vue arbitraire adopté. Dans leurs actions ou leurs effets, le mouvement brownien, dans un volume sphérique VA au cours du temps tg, de mise en place d'une organisation spatiale (loi de puissance $\mathbf{V A}=\mathbf{f}(\mathbf{t g})$ d'exposant $\mathbf{3} / \mathbf{2}$ ), ou le décours temporel (loi de puissance $\mathbf{t g}=\mathbf{f}(\mathbf{V A})$ d'exposant $\mathbf{2 / 3}$ ) et les phénomènes instantanés d'échanges à la surface d'une sphère (rapport de la surface au volume d'exposant $2 / 3$, ou du volume à la surface d'exposant 3/2) sont équivalents (loi systémique constructale). VA et tg sont des limitations de l'endophysiotope imposées à la fois par les limites de la capacité d'accueil de l'écoexotope et de la capacité d'être accueilli de l'endophysiotope, et tg est autant une limitation de VA que VA est une limitation de tg.

\section{N. Le mouvement brownien, loi de puissance 3/2, processus structural, constitutif et effet de notre Univers ?}

La loi de puissance ${ }^{26} \mathbf{V}_{\mathbf{A}}{ }^{2}=\mathbf{C} . \mathbf{t}_{\mathrm{g}}{ }^{3}$ qui relie le temps et l'espace à tous les niveaux d'organisation de l'Univers (niveau $\boldsymbol{i}+7)^{27}$ est semblable à la troisième loi de Kepler, caractéristique des systèmes stellaires (sous-systèmes de niveau $i+4$ ), sauf que le coefficient est $3 / 2$ et non $2 / 3$ (figure 6 ). C'est-à-dire que le temps y joue le rôle de l'espace et l'espace celui du temps (figure $3 a)$.

Cette loi $\mathbf{V}_{\mathrm{A}}=$ C.t. $_{\mathrm{g}}{ }^{3 / 2}$ se répète de façon fractale, pour le Tout, l'Univers, et pour ses parties (figure 3b), ce qui suggère que l'Univers pourrait être une répétition d'emboîtements (et de juxtapositions), la "sommation" d'une série d'événements de même loi de puissance $3 / 2 .{ }^{28}$ Sans faire appel à aucune loi thermodynamique, on "tombe" indirectement sur un phénomène thermodynamique fondamental de notre Univers : le mouvement brownien. ${ }^{29}$

Le mouvement brownien est lui-même auto-similaire.

On peut remarquer que $V_{A}{ }^{2 x 1 / 3}=C . t_{g}$ et que $2 / 3=1 /(3 / 2)$ indépendamment des unités dimensionnelles.

$2 / 3$ est souvent une valeur seuil dans les phénomènes de percolation (comme les incendies de forêt).

1/3 est la limite d'une série convergente de puissances de 2 évoquant la série des divisions d'un soussystème (par exemple une cellule initiale, niveau $i$ ) qui donne naissance à un système adulte (par exemple un organisme méta-cellulaire, niveau $i+1$ ) au cours de la phase de croissance de son cycle de développement [47]. Au niveau de l'Univers [34], la pente de la droite de régression entre la distance d'une source lumineuse et le décalage des longueurs d'onde reçues vers le rouge, qui permet la détermination expérimentale de la constante cosmologique de Hubble, est aussi de 2/3 .

26 Où $\mathbf{C}$ est une constante arbitraire qui dépend du système d'unités (figure 3) et du type de représentation (figure 6).

27 avec $\mathbf{V}_{\mathbf{A}}$ volume du système à l'état adulte (à la fin de la phase de croissance, ayant acquis la capacité reproductrice, typique de l'état adulte)

et $t_{\mathrm{g}}$ temps d'atteinte de l'état adulte (durée de la phase "larvaire“ de croissance, temps séparant 2 générations successives)

28 La "somme" (le Tout) étant globalement à la fois plus et moins que la somme (la sommation) de ses parties.

29 Le mouvement brownien est un mouvement aléatoire de particules qui ne sont soumises à aucune autre interaction que les chocs. 


\section{CONCLUSION}

Tous les systèmes vivants sont modulaires, organisés par emboîtements et juxtapositions de soussystèmes pré-existants. Cette modularité, structurale et fonctionnelle, permet la mise en place de niveaux hiérarchisés, dont les interactions, structurales et fonctionnelles, obéissent à des lois de puissance. Des contraintes géométriques, comme le confinement, sont suffisantes pour déclencher un comportement collectif global contraignant le comportement individuel local $[43,46]^{30}$. Cette course évolutive ${ }^{31}$ est un "matryoshka race" [63] qui, par itération, rend les endophysiotopes de plus en plus dépendants des endophysiotopes et des écoexotopes qu'ils internalisent (figure 3) et de plus en plus indépendants des écoexotopes qu'ils partagent avec d'autres formes de vie externes. La structure en éponge de l'Univers (figure 6) est totalement virtuelle. C'est la simulation numérique qui permet de la représenter à partir d'observations dans certaines circonstances. Aucun objet ne nous apparaît tel qu'il est à l'instant où on le regarde mais tel qu'il était lorsqu'à été émise la lumière qui nous renseigne sur lui. L'expansion de l'Univers est à la fois spatiale et temporelle (figure 3). Regarder loin dans l'Univers c'est regarder loin dans son passé. Nous n'avons accès à la réalité de l'Univers qu'à travers les modèles physico-mathématiques avec lesquels nous simulons les observations qui nous permettent de le décrire $[66,67]$. C'est toujours une image composite, hors de nos sens, une superposition d'observations perceptibles et imperceptibles (pour nous, mais par pour nos instruments) re-fabriquées à partir de simulations numériques d'équations issues de modèles et de théories explicatives et prédictives, qui changent avec l'évolution des observations, des outils et des modèles [25, 30].

À la fois consommateur d'écosystèmes, qui disparaissent à cause de lui, et producteur d'écosystèmes nouveaux qui n'existeraient pas sans lui, à la fois prédateur et espèce clé-de-voûte, l'homme bouleverse les niveaux d'organisation et d'intégration du vivant [31,32]. Pourtant il n'est qu'une forme de vie comme une autre, un support, un abri ou une proie, un écosystème d'accueil pour d'autres formes de vie, plus anciennes ou plus récentes, plus robustes ou plus résiliantes, que la sienne [11,13]. Se cacher, s'échapper, résister, se défendre, ou attaquer, pour manger et ne pas être mangé, présente toujours des avantages et des inconvénients (tableau 1). A tout niveau d'organisation, quelle que soit la stratégie de survie, elle représente un coût en matière et en énergie qui entraîne une réduction des potentialités de croissance, et qui doit être à nouveau payé à chaque génération. Quelle que soit la stratégie de survie, résister, tolérer, supporter, rejeter, coopérer ou se transformer, la plus performante est celle qui évite les situations d'escalade de la violence et qui permet de faire d'une pierre deux coups. Survivre c'est transformer des inconvénients en avantages et éviter que des avantages deviennent des inconvénients. Seules survivent, et se survivent, les Associations à Avantages et Inconvénients Réciproques et Partagés, qui rendent les partenaires toujours plus dépendants les uns des autres, indissociables, mais toujours plus indépendants de leur écoexotope de survie. Elles permettent d'accroître la capacité d'être accueilli de l'endophysiotope quand la capacité d'accueil de l'écoexotope diminue. ${ }^{32}$

30 "Collective behavior, through the emergence of complex migration patterns over scales larger than those of the individual actors constituting a system, plays a pivotal role in regulating various biological systems processes, such as gastrulation (movement), morphogenesis (reaction to stimulation) and tissue organization. Cell density (crowding), strength of intercellular adhesion (cohesion) and boundary conditions imposed by extracellular matrix proteins (constraints) are together involved in regulating the emergence of collective behavior within epithelial cell sheets. The geometrical confinement of cells into well-defined circles induces a persistent, coordinated and synchronized rotation of cells that depends on cell density. The speed (X1) of such large-scale movements slows down as the density (X2) increases (X1.X2=k). Such collective behavior depends on the size of the micro-patterned circles: a rotating motion of the overall cell population in the same direction is observed for sizes up to $200 \mu \mathrm{m}$. The rotating cells ( $\boldsymbol{i}$ level) move as a solid body, like an organism ( $\boldsymbol{i}+\boldsymbol{1}$ level), with a uniform angular velocity. This upper limit leads to length scales that are similar to the natural correlation length observed for unconfined epithelial cell sheets. This behavior is strongly altered in cells that present a down-regulation of adherens junctions and in cancerous cell types." [adapted from 46].

31 "Mutually beneficial associations between individuals ( $i+1$ level) of different species, called mutualistic symbioses, have enabled major ecological innovations and underlie some of the major transitions $(\boldsymbol{i}+\boldsymbol{2}$ level) in evolution. The ancestor of green plants domesticated endosymbiotic photosynthetic bacteria, today's chloroplasts, for carbon fixation. This association greatly increased the habitat of these photosynthetic bacteria from the sea to terrestrial ecosystems. However, the colonization of land by plants required an additional symbiotic association, with fungal root symbionts that facilitate nutrient uptake. Despite being unrelated, free-living algae and fungi can learn to help one another out, like in Lichen ancestors. Today the green alga Chlamydomonas reinhardtii in CO2-restricted environments and the yeast Saccharomyces cerevisiae depend on one another for the metabolic production of $\mathrm{CO} 2$, which is provided by the yeast as it consumes glucose and is needed by the alga, and ammonia, which conversely can be made from nitrite by the alga and then used by the yeast. This dependence is seen under a broad range of environmental conditions. Similar tests between other Chlamydomonas and fungal species revealed the ability to create a phylogenetically broad range of mutualisms." [adapted from 2]

32 "Mutualistic symbioses shape the evolution of species and ecosystems and catalyze the emergence of biological complexity. An obligate mutualism between the yeast Saccharomyces cerevisiae and the alga Chlamydomonas reinhardtii -eukaryotes with very different life historiescan arise spontaneously in an environment requiring reciprocal carbon and nitrogen exchange. This capacity for mutualism is phylogenetically broad, extending to other Chlamydomonas and fungal species. The spontaneous association of Chlamydomonas algal cells physically interacting with filamentous fungi demonstrate that under specific conditions, environmental change induces free-living species to become obligate mutualists and establishes a set of experimentally tractable, phylogenetically related, synthetic systems [adapted from 53]. 
- première règle: "pour survivre, il faut manger et ne pas être mangé".

Mais, tôt ou tard, il est impossible de ne pas être mangé !

Car tout système vivant est une chaîne alimentaire et il appartient à au moins une chaîne alimentaire.

Survivre c'est d'abord manger et ne pas être mangé.

- deuxième règle: "la croissance est le préalable à la reproduction".

il faut survivre, pour croître, et croître pour "se survivre", voire se multiplier.

Survivre n'a qu'un but "se survivre" : permettre la survie de sa forme de vie.

La vie naît et renaît du flux de matière et d'énergie qui la traverse.

Tous les systèmes vivants de notre "biosphère" sont interdépendants.

- troisième règle : quelle que soit la situation, "il n'y a jamais d'avantages sans inconvénients".

Survivre c'est "transformer les inconvénients en avantages

et éviter que les avantages deviennent des inconvénients".

- quatrième règle : dans tout système vivant, "les parties et le tout sont indissociables".

La survie de l'un (l'acteur local) passe par celle de l'autre (l'acteur global) et réciproquement.

"Pour qu'un acteur survive, il faut d'abord que tous les autres survivent et réciproquement."

Des inconvénients pour un acteur sont des avantages pour un autre et réciproquement.

- cinquième règle : la mise en place de réseaux d'interactions est le moteur de l'évolution.

A long terme, "seules survivent et se survivent les associations à avantages et inconvénients réciproques et partagés." http://www.armsada.eu

Au cours de l'évolution, c'est l'intégration à l'écoexotope de survie qui "actualise" des choix d'endophysiotopes, d'organisation et d'intégration temporairement durables.

Pour survivre et se survivre, "il faut être au bon endroit au bon moment" et "ne pas être au mauvais endroit au mauvais moment", à tout moment "il faut avoir eu de la chance et avoir encore de la chance suffisemment longtemps".

2 Les contraintes de survie de l'espace-temps-action des systèmes vivants. (Bricage 2007).

- première contrainte : tout système est une unité indissociable d'espace, de temps et d'action $(1,2)$. - deuxiéme contrainte : pour survivre et se survivre un système doit "réaliser la bonne action, au bon endroit, au bon moment" (3). C'est l'existence, à la fois, d'une organisation -structurale et fonctionnellemodulaire (I'endophysiotope) et de possibilités d'expression de choix qui permettent l'expression contingente d'une "praxis" en adéquation avec l'environnement naturel \& culturel (nature \& nurture) de survie (l'écoexotope).

- troisiéme contrainte : tout comportement de tout système vivant (4) est "à la fois plus et moins" que la somme ou/et le produit (5) des potentialités et des interactions (6) entre l'endophysiotope et l'écoexotope (7). - quatriéme contrainte : Survivre et se survivre c'est d'abord "connaitre et re-connaitre" les limites et les limitations (8) de son écoexotope (tope: espace-temps, exo : externe, éco : d'habitation et d'action, son "umwelt" structural \& fonctionnel) de survie et aussi les limites et les limitations de son endophysiotope (tope : espace-temps, endo : interne, physio : de fonctionnement et d'action, son "innerwelt" structural \& fonctionnel) $(9,10)$.

- cinquiéme contrainte : A long terme, "seules survivent et se survivent les associations à avantages et inconvénients réciproques et partagés." http://www.armsada.eu

Tableau 1. "survivre et se survivre" : règles et contraintes.

Adapté d'après [10], [11], [12], [13], [14], [17], [18], [20], [21], [25], [30].

La terre n'appartient pas à l'humanité, c'est l'humanité qui appartient à la terre...

Un développement durable, permettant de survivre aux hasards du milieu, est impossible, en donnant la force au faible et en anéantissant le fort..., il est impossible de permettre la co-existence en favorisant la lutte...

Tout compromis a un coût.

Le tout est espaces et temps, le tout est objets et réseaux $x^{33}$, le tout est pluri-actions, inter-actions et rétro-actions.

33 Quel que soit le niveau d'organisation, la mise en place du réseau s'effectue par percolation [38] en "obéissant" à une stratégie "eulerienne“ [63]. La mise en place d'un espace-temps eulerien permet de répartir "également" (dans l'espace) et "à tour de rôle" (dans le temps) les avantages et les inconvénients entre les acteurs d'un même Tout [22,23]. 
Plusieurs ne forment plus qu'un. L'avantage est énorme, mais le coût est aussi énorme [12]. Chacun pour survivre doit d'abord permettre la survie de l'autre, éventuellement à l'encontre de sa propre survie [13, 14]. Les parties et le Tout fonctionnent interdépendamment : "un pour tous et tous pour un" (tableau 1) [15, 19, 22].

L'aspect vivant des êtres réside à la fois dans les objets eux-mêmes et dans les propriétés des processus, de maintien ou de changement, auxquels ils contribuent, activement, par une succession incessante de changements dans l'activité des compartiments de leur organisation. Cette succession de changements, localement et globalement, obéit à une loi de croissance qui relie globalement de façon invariante, et indépendamment de l'échelle, le temps et l'espace, même si les courbes locales de croissance peuvent être différentes [58]. Quel que soit le niveau d'organisation, cette loi est présente dans les niveaux d'organisation adjacents, inférieurs et supérieurs (figure 3b). Elle "accompagne" la montée de complexité (figure 1.-6), universelle, "réalité indépendante,... réalité première par rapport à l'esprit humain". ${ }^{34}$

L'évaluation qualitative de la complexité est première. "Tout système complexe est à la fois plus et moins que la somme de ses parties, il faut d'abord mettre en oeuvre une approche réductionniste, du Tout au parties, puis une approche holistique, des parties au Tout. La connaissance du système est celles du Tout et des parties à la fois." Comparer 2 systèmes revient à comparer 2 algorithmes [41]. L'évaluation quantitative se fait en déterminant les types d'opérations (complexité d'action) et le nombre d'opérations (complexité temporelle) qu'ils requièrent, et la place nécessaire pour garder en mémoire les informations (complexité spatiale) [37]. Cet espace-temps-action dépend de l'état initial du système. La "complexité au pire", la plus encombrante en espace-temps-actions, "délimite" le système.

Toute structure organisée est maintenue par des interactions opposées. Le tout est moins que la somme de ses parties et l'assemblage des parties dans le tout est irréversible. Tout équilibre est continuellement déconstruit et reconstruit par des rétro-actions (loi systémique constructale). Tôt ou tard la croissance s'arrête. La survie optimale va à l'encontre d'une stratégie de croissance maximale. Une croissance minimale, parcimonieuse, est nécessaire pour acquérir la capacité de se survivre. Pour sa seule survie, l'homme détourne à son profit, toute activité vivante [32] ! Tout être vivant, l'homme y compris, peut maintenant être modifié, par l'homme, dans son fonctionnement. En asservissant la nature et la vie, pour la fabrication d'hommes, l'homme libère les dangers contenus [26] dans les associations symbiotiques, agressées par les activités humaines. Des virus présents bien avant l'homme (comme le virus Ebola) se propagent, à la suite de la coïncidence de séries de facteurs facilitant l'infection, par des hôtes intermédiaires. Quelle que soit la maladie, d'origine virale externe ou interne, il existe toujours des individus sains, sans symptôme, et vecteurs du virus [22, 36, 38, 39].

\section{REFERENCES}

[1]

$[2]$ 209-240, October 2011 C. Bessa-Gomes \& al., "Mating behaviour influences extinction risk: insights
comparative analysis of avian extinction risk.", Ann. Zool. Fennici, Vol. 40, pp. 231-245, April 2003.

K. Aagaard \& al., "The placenta harbors a unique microbiome.", Sci. Transl. Med., Vol. 6, no 237, 237ra65, May 2014. D.K. Aanen \& T. Bisseling, "The birth of cooperation.“, Science, Vol. 345, no 6192, pp. 29-30 July 2014.

E. Almaas \& A.-L. Barabasi, "Power laws in biological networks.“, In Power Laws, Scale-Free Networks and Genome Biology, E.V. Koonin, Y.I. Wolf \& G.P. Karev eds, Intelligence Unit Book Series, Eurekah.com \& Springer, Korea, pp. 1-11, March 2006.

A. Bejan \& S. Lorente, "The constructal law and the evolution of design in nature." Physics of Life Reviews, Vol. 8, pp. rapports entre les êtres vivants et leur milieu de vie: exemples d'actions des facteurs du milieu sur les êtres vivants. Bull. A.A.S.N.S., Vol. 55, pp. 17-41, juin 1976.

[7] P. Bricage, "Pigmentation and soluble peroxidase isozyme patterns of leaves of Pedilanthus tithymaloides L. variegatus as a result of daily temperature differences.“, Plant Physiology, Vol. 69, pp. 668-671, June 1982.

[8] P. Bricage, "Isoperoxidases, markers of surrounding and physiological changes, in situ in leaves and in vitro in calli of Pedilanthus tithymaloides L. variegatus: cell compartmentation and polyfunctionality, control of activity by phenols, specific roles.", In Molecular \& Physiological Aspects of Plant Peroxidases, H. Greppin \& al., eds, Geneva, Switzerland, pp. 261-265, June 1986.

[9] P. Bricage, "Les Caractéristiques des Organismes Vivants.", Fac. Sciences, Univ. Pau, UPPA, APIDS, 44 p., mars 1991.

[10] P. Bricage, "La survie des systèmes vivants.“, atelier MCX20 Prendre soin de l'homme, CHG Pau, 2 p., octobre 1998, CC-License, http://web.univ-pau.fr/ $\sim$ bricage/VivantPauMCX20.pdf

[11] P. Bricage (2000a), "La survie des organismes vivants.“, atelier AFSCET systémique \& biologie, Fac. Médecine des Saints Pères, Paris, 44 p., février 2000, CC-License, http://www.afscet.asso.fr/SURVIVRE.pdf

[12]

P. Bricage (2000b), "La nature de la violence dans la nature : Déterminismes écologique, physiologique et génétique de l'adaptation aux changements aux différents niveaux d'organisation des systèmes végétaux.", La Violence. Colloque AFSCET, Andé, France, 7 p., juin 2000, CC-License, http://www.afscet.asso.fr/pbviolW98.pdf

34 B. d'Espagnat, “À la recherche du réel.” Bordas, Paris, 194 p., février 1993. 
P. Bricage (2000c), "Systèmes biologiques : le jeu de la croissance et de la survie. Quelles règles ? Quelles décisions ? Quels bilans? La décision systémique : du biologique au social. Atelier AFSCET, Paris, Institut International d'Administration Publique, 6 p., décembre 2000, CC-License, http://www.afscet.asso.fr/JdViel.pdf

[14]

P. Bricage (2001a), "La nature de la décision dans la nature ? Systèmes biologiques: production, consommation, croissance et survie. Quelles règles? Quels degrés d'exigence ? Quels bilans ?“, La décision systémique : du biologique au social., Colloque AFSCET, Andé, 16 p., juin 2001, CC-License, http://www.afscet.asso.fr/Decision.pdf

[15] P. Bricage (2001b), "A new evolutionary paradigm : the Associations for the Mutual Sharing of Advantages and of Disadvantages.", In The creation of a sustainable society depends on Innovative Systems Thinking. 100th Anniversary of Karl Ludwig von Bertalanffy's International Conference on Systems Thinking "Unity through Diversity", Vienna, Austria, 1 p., April 2001.

[16] P. Bricage (2001c), "Les communautés en Vic-Bilh : associations matrimoniales et patrimoine associatif." Journées européennes du patrimoine. Centenaire des Associations Loi 1901, Bull. Association ALBA, Vol. 17, 4 p., septembre 2001.

[17] P. Bricage(2001d), "Pour survivre et se survivre, la vie est d'abord un flux, ergodique, fractal et contingent, vers des macro-états organisés de micro-états, à la suite de brisures de symétrie.“ atelier AFSCET systémique \& biologie, Institut International d'Administration Publique, Paris,11 p., décembre 2001, CC-License, http://www.afscet.asso.fr/ergodiqW.pdf

[18] P. Bricage (2002a), "Héritage génétique, héritage épigénétique et héritage environnemental : de la bactérie à l'homme, le transformisme, une systémique du vivant. "Évolution du vivant et du social : Analogies et différences. Colloque AFSCET, Andé, 20 p., juin 2002, CC-License, http://www.afscet.asso.fr/heritage.pdf

[19] P. Bricage (2002b), "The Evolutionary Shuttle of the Living Systems.“, UES-EUS Congress, Hersonissos, Creta, Greece, Res. Systemica, Vol. 2, 6 p., October 2002, CC-License, http://www.afscet.asso.fr/resSystemica/Crete02/Bricage.pdf

[20] P. Bricage, "Organisation, intégration et espace-temps des systèmes vivants." Intégration du vivant et du social : Analogies et différences, Colloque AFSCET, Andé, 31 p., juin 2003, CC-License, http://www.afscet.asso.fr/pbAnde03.pdf

[21] P. Bricage (2005a), "Le langage du vivant : plurilinguisme, transfrontaliérité et associativité." Congrès national ANLEA, UPPA, Pau, 9 p., mars 2005, CC-License, http://www.abbayeslaiques.asso.fr/BIOsystemique/ANLEA05PauPB.pdf

[22] P. Bricage (2005b), "The Cell originated through Successive Outbreaks of Networking and Homing into Associations for the Mutual and Reciprocal Sharing of Advantages and of Disadvantages, between the Partners, with a Benefit only for their Wholeness.“, UES-EUS Congress, Paris, France, Res. Systemica, Vol. 5, 11 p., September 2005, CC-License, http://www.afscet.asso.fr/resSystemica/Paris05/bricage3.pdf

[23] P. Bricage (2005c), "The Modelling of the Time Modularity of the Living Systems: the Time Delay, the Time Duration, the Time Lag, and the Rhythms.", UES-EUS Congress, Paris, France, Res. Systemica, Vol. 5, 11 p., September 2005, CC-License, http://www.afscet.asso.fr/resSystemica/Paris05/bricage2.pdf

[24] P. Bricage, "Les systèmes agoantagonistes : l'origine endosyncénotique de la cellule.” UES-EUS Congress, Paris, In La Gouvernance dans les Systèmes. Collectif AFSCET, Polimetrica, Milan, pp. 67-72, mai 2007, CC-License, http://www.afscet.asso.fr/resSystemica/Paris05/bricage3.pdf \& http://www.afscet.asso.fr/resSystemica/Paris05/bricage.pdf

P. Bricage (2008a), "La démarche scientifique expérimentale : un langage systémique. Mise en évidence d'une phase critique d'apprentissage." Systémique et langage, Colloque international AFSCET, Andé, 30 p., juin 2008, CC-License, http://www.afscet.asso.fr/Ande08/pbAnde08ExpSci.pdf

[26] P. Bricage (2008b), "Cancer is a breaking of the cell's ARMSADA through an aggression that results in a lack of nonautonomy.“, UES-EUS Congress, APOCOSIS, Lisboa, Portugal, Res. Systemica, Vol. 6, 8 p. \& 26 p., December 2008, CC-license, http://www.afscet.asso.fr/resSystemica/Lisboa08/bricagel.pdf, $\quad$ http://www.afscet.asso.fr/resSystemica/Lisboa08/bricageCancer.pdf, http://tinyurl.com/pbcancerlisboa \& http://www.afscet.asso.fr/resSystemica/Lisboa08/pbdiscussion.pdf

[27] P. Bricage, "L'évolution créatrice : métamorphoses et phylotagmotaphologie du vivant. L'évolution du Vivant a une direction. Comment est-elle orientée ?" Colloque http://www.teilhard.org " 150 ans après Darwin, 70 ans après Teilhard : Lire l'évolution." Centre de Sèvres, Paris, 109 p., décembre 2009, CC-License, http://hal.archives-ouvertes.fr/hal-00423730/fr

[28] P. Bricage (2010a), "Approche systémique de l'évolution du vivant.“, Teilhard Aujourd'hui, Vol. 33, pp. 31-39, mars 2010, CC-License, http://hal.archives-ouvertes.fr/docs/00/42/37/30/PDF/phylotagmotaphologie.pdf

[29] P. Bricage (2010b), "Sémiologique graphique de l'espace-temps-action du vivant : bilan épistémologique et praxéologique de sa modélisation systémique, co-déterminisme global, prédictibilité locale et imprédicativité glocale." Vers une nouvelle systémique ? Colloque AFSCET, Andé, 34 p., mai 2010, CC-License, http://www.afscet.asso.fr/Ande10/pbETAvivant10.pdf

[30] P. Bricage (2011a), "Engineering Health and Social Sciences Curriculum: Governance of the Pedagogy, Pedagogy of the Governance.", In Social Responsibility: Holistic Education for Holistic Behaviour, IRDO, Maribor, Slovenia., 7 p., March 2011, CCLicense, http://tinyurl.com/HSSengineering

[31] P. Bricage (2011b), "La semi-autonomie du vivant : pour que l'un survive, il faut d'abord que l'autre survive, et réciproquement“., UES-EUS Congress, Crises et réponses systémiques, Bruxelles, Belgique, 28 p., septembre 2011, CC-License, http://www.armsada.eu/files/pbARMSADA.pdf

[32] P. Bricage (2011c), “About Man Interventions in the Living Networks: Modelling with a Qualitative Animated Semiological Holistic Point of View, a Systemic Approach, in an Holistic Way of Education to Explain The Issues of the Fighting Steps and the Escalade of Violence between Mankind and the Wild.", Mankind IASCYS workshop, 25 p., September 2011, CC-License, http://www.armsada.eu/files/pbManSERqash.pdf

[33] P. Bricage (2013a), "Les principes organisateurs d'émergence des systèmes vivants.“, Maison des Arts et Métiers, Paris, 62 p., [web, 12 février 2013], CC-License, http://hal.archives-ouvertes.fr/docs/00/82/98/15/PDF/pbemergence2013 texte.pdf

[34] P. Bricage (2013b), "Mobilisation de la matière et de l'énergie, et croissance, aux différents niveaux d'organisation des systèmes vivants. Principes organisateurs d'émergence des systèmes vivants.", Thermodynamique du changement. Aspects systémiques, Journées annuelles AFSCET, Andé, 63 p. [web, 2 juin 2013], CC-License, http://www.afscet.asso.fr/Ande13/pbAnde2013.pdf 
P. Bricage (2013c), "Time Management by Living Systems: Time Modularity, Rhythms and Conics Running Calendars. Methodology, Theory and Applications." System Research \& Behavioral Science, Vol. 30, Issue 6, pp. 677-692, March 2013.

P. Bricage (2014a), "Agoantagonisme, rétrocession et émergence : organisation des systèmes vivants, éducation à la systémique, santé et sociétalité.“, In Hommage à Emmanuel Nunez, AFSCET-AEIS, CNAM, Paris, 15 février 2014, Res-Systemica, Vol. 10, article 11, 19 p., [web, 19 mai 2014], CC-License, http://web.univ-pau.fr/ bricage/ResSystemica2014nunez.pdf

[37] P. Bricage (2014b), "Associations for the Reciprocal and Mutual Sharing of Advantages and DisAvantages (ARMSADA). A Fruitful Paradigm.“, Social and Environmental Responsibility, IASCYS Workshop, EMCSR, Vienna, 30 p., April 2014, CC-License, http://armsada.eu/pbARMSADAemcsr2014.pdf

[38] P. Bricage (2014c), "Local versus global \& individual versus whole. Competition between \& within living systems. ARMSADA emergence and breaking.“, UES-EUS Congress, Valencia, Spain, 15 p., October 2014, Advanced On Line Publication July 2014, CC-License, http://web.univ-pau.fr/ bricage/files/bricage_s02-5c-paper.pdf

[39] P. Bricage (2014d), "Survival Management by Living Systems. A General System Theory of the Space-Time Modularity and Evolution of Living Systems.", 2 p., Advanced On Line Publication, March 2014, CC-License, http://www.wccs14.org/

[40] B. Buhai, A Kühnle \& R. Kimmich, "NMR flow mapping and computational fluid dynamics in Ising-correlated percolation model objects.“, New J. Phys. Vol. 7, pp. 157- 178, March 2005.

[41] J.L. Chabert, "Les algorithmes.“, In Les Mathématiques en 14 mots-clés. La Recherche H.S., Vol. 4, Spécial Mathématiques, pp. 64-69, novembre 2008.

[42] J. Collier, "Regulation of chromosomal replication in Caulobacter crescentus.“, Plasmid Vol. 67, pp. 76-87, mars 2012.

[43] M. Deforet \& al., "Emergence of collective modes and tri-dimensional structures from epithelial confinement." Communications, Vol. 5, no 3747, Nature Communications ISSN (online): 2041-1723, July 2014.

[44] A.K. Dickerson \& al., "Wet mammals shake at tuned frequencies to dry.", J. R. Soc. Interface, Vol. 9, pp. 110-123, December 2012.

[45] P. Dodds \& al., "Re-Examination of the 3/4-Law of Metabolism.", J. Theor. Biol., Vol. 209, pp. 9-27, 2001. (Physical Review Letter, Vol. 91, p. 247901, March 2010, Nature 464, 753-756, April 2010.)

[46] K. Doxzen \& al., "Guidance of collective cell migration by substrate geometry.", Integrative biology: quantitative biosciences from nano to macro Vol. 5, pp. 1026-1035, June 2013.

[47] F. Dress \& M. Mendès France, "La suite des puissances de 3/2.“, La Recherche, $\mathrm{n}^{\circ} 346-\mathrm{n}^{\circ}$ spécial hors série- : Grandes et petites énigmes Mathématiques., pp. 34-37, octobre 2001.

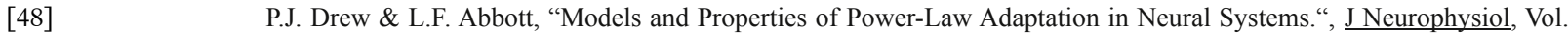
96, pp. 826-833, April 2006.

[49] S.R. Dunbar, "The definition of Brownian Motion and the Wiener Process.", In $\underline{\text { Stochastic Processes and Advanced }}$ Mathematical Finance., Lincoln, Nebraska, USA, 17 p., February 2014, http://www.math.unl.edu

[50] K. Flärdh, "Cell polarity and the control of apical growth in Streptomyces.“, Curr. Opin. Microbiol., Vol. 13, pp. 758765, December 2010.

[51] K. Goh, B. Kahng \& D. Kim, "Graphical Analysis of Biocomplex Networks and Transport Phenomena.", In Power Laws, Scale-Free Networks and Genome Biology, edited by E.V. Koonin, Y.I. Wolf and G.P. Karev, Intelligence Unit Book Series, Eurekah.com and Springer Science+Business Media, Korea, pp. 12-24, March 2006.

[52] K.J. Greenlee \& al., "Synchrotron imaging of the grasshopper tracheal system: morphological and physiological components of tracheal hypermetry.“, Am J Physiol Regul Integr Comp Physiol, Vol. 297, pp. 1343-1350, August 2009.

[53] E.F.Y. Hom \& A.W. Murray, "Niche engineering demonstrates a latent capacity for fungal-algal mutualism.“, $\underline{\text { Science, }}$ Vol. 345, no. 6192, pp. 94-98, July 2014

[54] A.J. Hulbert \& al., "Life and Death: Metabolic Rate, Membrane Composition, and Life Span of Animals." Physiol. Rev, Vol. 87, pp. 1175-1213, October 2007.

[55] G.L. Hunter \& al., "Tracking rotational diffusion of colloidal clusters.“ Optics Express, Vol. 19, Issue 18, pp. 1718917202, August 2011.

[56] J.-P. Kahane, "Le mouvement brownien et son histoire, réponses à quelques questions", Images des Mathématiques, CNRS, octobre 2006, http://images.math.cnrs.fr/Le-mouvement-brownien-et-son.html

[57] B.F. Lang \& al., "Massive programmed translational jumping in mitochondria." PNAS, Vol. 111, Issue 16, pp. 59265931, April 2014.

[58] A.H. Lee \& S. Werning, "Sexual maturity in growing dinosaurs does not fit reptilian growth models." $\underline{\text { PNAS, }}$ Vol. 105, Issue. 2, pp. 582-587, January 2008.

[59] Q.Q. Lee \& al., "Emasculation: gloves-off strategy enhances eunuch spider endurance.”, Biology Letters, Vol. 8, Issue 5, pp.733-735, June 2012 .

[60] D. Lloyd, S. Legendre \& P. Harremöes, "Evolutionary Entropy: A Predictor of Body Size, Metabolic Rate and Maximal Life Span.“, Bulletin of Mathematical Biology, Vol. 71, pp. 800-818, May 2009.

[61] J.-P. Luminet, "La topologie cosmique“. In Les mystères du cosmos du big bang aux trous noirs. Dossier HS Pour La Science, Vol. 83, pp. 92-99, avril 2014.

[62] C. Michaut, "La liaison chimique." In La Physique en 18 mots-clés. La Recherche H.S. Vol. 1, Spécial Physique, pp. 64-69, septembre 2005. 
F. Miniati, "The Matryoshka run: a Eulerian Refinement strategy to study the statistics of turbulence in virtualized cosmic structures.“, Astrophysical J., Vol. 782, Issue 1, pp. 21, April 2014.

[64]

F. Perrin, "Etude mathématique du mouvement Brownien de rotation," Ann. Sci. Ec. Normale Super. Série 3, Vol. 45, pp. 1-51, janvier 1928.

[65] O. Rios-Cardenas, "Patterns of Parental Investment and Sexual Selection in Teleost Fishes: Do They Support Bateman's Principles?", Integrative and Comparative Biology, Vol. 45, Issue 5, pp. 885-894, December 2005.

[66] B. Romney (2014a), "Personne n'a jamais vu un électron.“, La Recherche, Vol. 489, pp. 35- 38, juillet 2014.

[67] B. Romney (2014b), "Cosmos : voir au-delà des images.", La Recherche, Vol. 489, pp. 42- 47, juillet 2014.

[68] M. Thomas-Orillard \& S. Legendre, "Virus C de la drosophile et dynamique d'une population hôte.”, C.R. Acad. Sci. Paris, Life Sciences, Vol. 319, pp. 615-621, juin 1996.

[69] R.L. Trivers, "Parental investment and sexual selection.“, In B. Campbell (ed.), Sexual selection and the descent of man., Aldine, Chicago, pp. 136-179, July 1972.

[70] R.L. Trivers, "Social evolution.", Benjamin Cummings, Menlo Park, September 1985.

[71] A. Tzur \& al., "Cell growth and size homeostasis in proliferating animal cells.“, $\underline{\text { Science }}$, Vol. 325, pp. 167-171, July 2009. 